\title{
NONLOCAL INTERACTIONS BY REPULSIVE-ATTRACTIVE POTENTIALS: RADIAL INS/STABILITY
}

\author{
D. BALAGUÉ ${ }^{1}$, J. A. CARRILLO ${ }^{2}$, T. LAURENT ${ }^{3}$ AND G. RAOUL $^{4}$
}

\begin{abstract}
We investigate nonlocal interaction equations with repulsive-attractive radial potentials. Such equations describe the evolution of a continuum density of particles in which they repulse each other in the short range and attract each other in the long range. We prove that under some conditions on the potential, radially symmetric solutions converge exponentially fast in some transport distance toward a spherical shell stationary state. Otherwise we prove that it is not possible for a radially symmetric solution to converge weakly toward the spherical shell stationary state. We also investigate under which condition it is possible for a non-radially symmetric solution to converge toward a singular stationary state supported on a general hypersurface. Finally we provide a detailed analysis of the specific case of the repulsive-attractive power law potential as well as numerical results.
\end{abstract}

\section{INTRODUCTION}

Nonlocal interaction equations are continuum models for large systems of particles where every single particle can interact not only with its immediate neighbors but also with particles far away. These equations have a wide range of applications. In biology they are used to model the collective behavior of a large number of individuals, such as a swarm of insects, a flock of birds, a school of fish or a colony of bacteria [46, 56, 57, 26, 27, 37, 24, 25, 16, 55, 6, 12, 5, 29, 15, 14, 13. In these models individuals sense each other at a distance, either directly by sound, sight or smell, or indirectly via chemicals, vibrations, or other signals. Nonlocal interaction equations also arise in various contexts in physics. They are used in models describing the evolution of vortex densities in superconductors [61, 53, 154, 42, 2, 1, 43, 31, 44]. They also appear in the modeling of dynamics of agglomerating particles in two dimensions (with loose links to the one-dimensional sticky particles system) [48]. They also appear in simplified inelastic interaction models for granular media [28, 20, 58, 41]. Going back to biology, nonlocal interaction equations arise also in the modeling of the orientational distribution of $\mathrm{F}$-actin filaments in cells [35, 38, 51].

In their simplest form, nonlocal interaction equations can be written as

$$
\frac{\partial \mu}{\partial t}+\operatorname{div}(\mu v)=0 \quad, \quad v=-\nabla W * \mu
$$

where $\mu(t, x)=\mu_{t}(x)$ is the probability or mass density of particles at time $t$ and at location $x \in \mathbb{R}^{N}, W: \mathbb{R}^{N} \rightarrow \mathbb{R}$ is the interaction potential and $v(t, x)$ is the velocity of the particles. We will always assume that the interaction potential $W(x)=k(|x|)$ is radial and $C^{2}$ - or $C^{3}$-smooth away from the origin, depending on the results. Typically the potentials we will consider have a singularity at the origin.

When the potential $W$ is purely attractive, i.e. $W$ is a radially symmetric increasing function, then the density of particles collapse on itself and converge to a Dirac Delta function located at the center of mass of the density. This Dirac Delta function is the unique stable 
steady state and it is a global attractor [18. The collapse toward the Dirac Delta function can take place in finite time if the interaction potential is singular enough at the origin and several works have been recently devoted to the understanding of these singular measure solutions [10, 8, 18, 9].

In biological applications however, it is often the case that individuals attract each other in the long range in order to remain in a cohesive group, but repulse each other in the short range in order to avoid collision [47, 49]. This lead to the choice of a radially symmetric potential $W$ which is first decreasing then increasing as a function of the radius. We refer to these type of potentials as repulsive-attractive potentials. Compared with the purely attracting case where solutions always converge to a single Delta function, nonlocal interaction equations with repulsive-attractive potentials lead to solutions converging to possibly complex steady states. As such, nonlocal interaction equations with repulsive-attractive potentials can be considered as a minimal model for pattern formation in large groups of individuals.

Whereas nonlocal interaction equations with purely attractive potential have been intensively studied there are still relatively few rigorous results about nonlocal interaction equations with repulsive-attractive potential. The $1 \mathrm{D}$ case has been studied in a series of works [33, 32, 52. The authors have shown that the behavior of the solution depends highly on the regularity of the interaction potential: for regular interaction, the solution converges to a sum of Dirac masses, whereas for singular repulsive potential, the solution remains uniformly bounded. They also showed that combining a singular repulsive with a smooth attractive potential leads to integrable stationary states. Pattern formation in multi-dimensions have recently been studied in [39, 60]. In these two works, the authors perform a numerical study of the finite particle version of (1) and show that a repulsive-attractive potential can lead to the emergence of surprisingly complex patterns. To study these patterns they plug in (1) an ansatz which is a distribution supported on a surface. This give rise to an evolution equation for the surface. They then perform a linear stability analysis around the uniform distribution on the sphere and derive simple conditions on the potential which classify the different instabilities. The various instability modes dictate toward which pattern the solution will converge. They also check numerically that what is true for the surface evolution equation also holds for the continuum model (1). In another recent work 34] the specific case where the repulsive part of the potential is the Newtonian potential and the attractive part is polynomial is analyzed showing the existence of radially compactly supported integrable stationary states. They also study their nonlinear stability for particular cases.

In this paper we focus primarily on proving rigorous results about the convergence of radially symmetric solutions toward spherical shell stationary states in multi-dimensions.

Definition 1 (Spherical Shell). The spherical shell of radius $R$, denoted $\delta_{R}$, is the probability measure which is uniformly distributed on the sphere $\partial B(0, R)=\left\{x \in \mathbb{R}^{N}:|x|=R\right\}$.

Given a repulsive-attractive radial potential whose attractive force does not decay too fast at infinity, there always exists an $R>0$ so that the spherical shell of radius $R$ is a stationary state as it will be remarked below. One need then to address the question of wether or not this spherical shell is stable. It is classical, see [3, 20, 59, 21], that the equation (1) is a gradient flow of the interaction energy

$$
E[\mu]=\frac{1}{2} \iint_{\mathbb{R}^{N} \times \mathbb{R}^{N}} W(x-y) d \mu(x) d \mu(y)
$$


with respect to the euclidean Wasserstein distance. Thus, stable steady states of (1) are expected to be local minimizers of the interaction energy. Simple energetic arguments will show that in order for the spherical shell of radius $R$ to be a local minimum of the interaction energy, it is necessary that the potential W satisfies:

(C0) Repulsive-Attractive Balance: $\omega(R, R)=0$,

(C1) Fattening Stability: $\partial_{1} \omega(R, R) \leq 0$,

(C2) Shifting Stability: $\partial_{1} \omega(R, R)+\partial_{2} \omega(R, R) \leq 0$,

where the function $\omega: \mathbb{R}_{+}^{2} \longrightarrow \mathbb{R}$ is defined by

$$
\omega(r, \eta)=-\frac{1}{\sigma_{N}} \int_{\partial B(0,1)} \nabla W\left(r e_{1}-\eta y\right) \cdot e_{1} d \sigma(y)
$$

$\sigma_{N}$ is the area of the unit ball in $\mathbb{R}^{N}, e_{1}$ is the first vector of the canonical basis of $\mathbb{R}^{N}$, $d \sigma$ denotes the volume element of the manifold where the integral is performed and $\mathbb{R}_{+}^{2}=$ $(0,+\infty) \times(0,+\infty)$. Condition (C0) simply guarantees that the spherical shell $\delta_{R}$ is a critical point of the interaction energy. We will see that if condition (C1) is not satisfied then it is energetically favorable to split the spherical shell into two spherical shells. Heuristically this indicate that the density of particles, rather than remaining on the sphere, is going to expand and occupy a domain in $\mathbb{R}^{N}$ of positive Lebesgue measure. If condition (C1) is not satisfied we will therefore say that the "fattening instability" holds. It can be easily checked that if $\omega(R, R)=0$, then $\partial_{1} \omega(R, R)$ is simply the value of the divergence of the velocity field on the sphere of radius $R$. So the fattening instability corresponds to an expanding velocity field on the support of the steady state. We will also see that if condition (C2) is not satisfied it is energetically favorable to increase or decrease the radius of the spherical shell. This instability will be referred as the "shift instability".

We now outline the structure of the paper and describe the main results. In the preliminary section, section 2, we derive (C0)-(C2) from an energetic point of view and we show that they correspond to avoiding the fattening and shift instability. We also study the regularity of the kernel $\omega$ defined by (2). A good understanding of the regularity of $\omega$ will be necessary for later sections. We also remind the reader of previous results from [11, 4] about well posedness of (1) in $L^{p}\left(\mathbb{R}^{N}\right)$. Section 3 is devoted to a detailed study of the fattening instability, both in the radially symmetric case and in the non-radially symmetric case. We first show that if condition $(\mathbf{C 1})$ is not satisfied then it is not possible for a radially symmetric $L^{p}$-solution to converge weakly-* as measures toward a spherical shell stationary state. We then investigate singular stationary states supported on hypersurfaces which are not necessarily spheres. Such steady states have been observed in numerical simulations [39, 60]. We show that if the divergence of the velocity field generated by such stationary state is positive everywhere on their support, then it is not possible for an $L^{p}$-solution to converge toward the stationary state in the sense of the topology defined by $d_{\infty}$. Here $d_{\infty}$ stands for the infinity-Wasserstein distance on the space of probability measures (see section 3 for a definition). We also show that if the repulsive-attractive potential $W$ is singular enough at the origin, for example $W(x) \sim-|x|^{b} / b$ as $|x| \rightarrow 0$ with $b \leq 3-N$, then the potential is so repulsive in the short range that solutions can not concentrate on an hypersurface, and this is independent of how attractive is the potential in the long range. To be more precise we show that for potentials with such a strong repulsive singularity at the origin, $L^{p}$ solutions can not converge with respect to the $d_{\infty}$-topology toward singular steady states supported on hypersurfaces. 
Whereas section 3 is devoted to instability results, section 4 is devoted to stability results. We show that if (C0) $-(\mathbf{C 2})$ hold with strict inequalities, then a radially symmetric solution of (1) which starts close enough to the spherical shell in the $d_{\infty}$ topology will converge exponentially fast toward it. Under additional assumptions on the potential we can also prove convergence with respect to the $d_{\alpha}$ topology, $\alpha \in[1,+\infty)$. In order for the stability results of section 4 to hold a certain amount of regularity on the solutions is necessary. Unfortunately

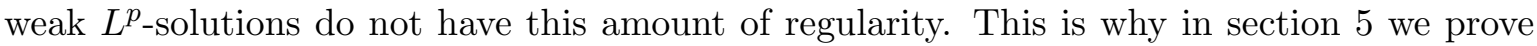
well posedness of classical $C^{1}$-solutions. This covers a gap in the existing literature which mostly considers weak solutions. The results of section 4 are true for this class of classical $C^{1}$-solutions. The aim of section 6 is to show examples of how to apply the general instability and stability theory in the case of power-law repulsive-attractive potentials:

$$
W(x)=\frac{|x|^{a}}{a}-\frac{|x|^{b}}{b} \quad 2-N<b<a .
$$

For this family of potentials, conditions (C0)-(C2) can be explicitly formulated in terms of $a$ and $b$, therefore leading to an explicit bifurcation diagram for the stability of the spherical shell in $\mathbb{R}^{N}$. Finally in the last section, section 7 , we perform numerical computations of radially symmetric solutions of (1) with power-law potential (3) and study their convergence toward spherical shell stationary state. Since a spherical shell is a highly singular function, it is challenging to perform such computations with traditional methods. This is why, following [36, 13, 22, 33, 32, rather than simulating (1) directly, we simulate the evolution of the inverse of the cumulative distribution of the radial measure associated to $\mu$. Since the inverse of the cumulative distribution of a spherical shell is a constant function, this approach has the virtue of smoothing the dynamics and this provides us with a robust numerical scheme. Our numerical simulations indicate the possible existence of integrable radial stationary states stable under radial perturbations in the parameter area corresponding to the fattening instability for power-law repulsive-attractive potentials, an issue that will be analysed elsewhere. This has already been proved in the particular case of $b=2-N$ and $a \geq 2$ in [34].

\section{Preliminary Section}

\subsection{Radially symmetric formulation of the equation.}

Definition 2 (Radial Measures). We denote by $\mathcal{P}^{r}\left(\mathbb{R}^{N}\right)$ the space of radially symmetric probability measures. If $\mu \in \mathcal{P}^{r}\left(\mathbb{R}^{N}\right)$ then $\hat{\mu} \in \mathcal{P}([0,+\infty))$ is defined by

$$
\int_{r_{1}}^{r_{2}} d \hat{\mu}(r)=\int_{r_{1}<|x|<r_{2}} d \mu(x) \quad \text { and } \quad \int_{0}^{r_{2}} d \hat{\mu}(r)=\int_{0 \leq|x|<r_{2}} d \mu(x)
$$

for all $0<r_{1}<r_{2}$. We endow this space with the standard weak-* topology.

Recall that $\delta_{R} \in \mathcal{P}^{r}\left(\mathbb{R}^{N}\right)$ stands for the spherical shell of radius $R$ (see Definition 1 ). The velocity field at point $x$ generated by a spherical shell of radius $R$ is given by $v_{R}(x)=$ $-\nabla W * \delta_{R}(x)$. Since $W$ is radially symmetric, then by symmetry there exists a function $\omega(r, \eta)$ such that

$$
v_{R}(x)=-\nabla W * \delta_{R}(x)=\omega(|x|, R) \frac{x}{|x|}
$$

and one can easily check that this function $\omega$ is defined by (2], see [8] for more details. Note also that if $\mu \in \mathcal{P}^{r}\left(\mathbb{R}^{N}\right)$ then it can be written as a sum of spherical shells, $\mu=\int_{0}^{+\infty} \delta_{\eta} d \hat{\mu}(\eta)$, 
and we conclude that

$$
-(\nabla W * \mu)(x)=-\int_{0}^{+\infty}\left(\nabla W * \delta_{\eta}\right)(x) d \hat{\mu}(\eta)=\int_{0}^{+\infty} \omega(|x|, \eta) d \hat{\mu}(\eta) \frac{x}{|x|} .
$$

Given $T>0, C\left([0, T] ; \mathcal{P}^{r}\left(\mathbb{R}^{N}\right)\right)$ denotes the set of continuous curves of radial measures where continuity is with respect to the weak-* convergence. We say that $\mu \in C\left([0, T] ; \mathcal{P}^{r}\left(\mathbb{R}^{N}\right)\right)$ is a radially symmetric solution of $(1)$ if $\hat{\mu} \in C([0, T] ; \mathcal{P}([0,+\infty))$ satisfies the one dimensional conservation law:

$$
\begin{aligned}
& \partial_{t} \hat{\mu}+\partial_{r}(\hat{\mu} \hat{v})=0 \\
& \hat{v}(t, r)=\int_{0}^{+\infty} \omega(r, \eta) d \hat{\mu}_{t}(\eta),
\end{aligned}
$$

in the distributional sense. We will now give conditions for the velocity field to be well-defined by studying the properties of the function $\omega$.

2.2. Regularity of the function $\omega(r, \eta)$. Let us remind that we assume that $W$ is radially symmetric and belongs to $C^{2}\left(\mathbb{R}^{N} \backslash\{0\}\right)$. The function $\omega(r, \eta)$ defined by $(2)$ is clearly $C^{1}$ away from the diagonal $\mathcal{D}=\{(r, r): r>0\}$. Moreover, the derivatives of $\omega$ are given by

$$
\partial_{1} \omega(r, \eta)=-\frac{1}{\sigma_{N}} \int_{\partial B(0,1)} \frac{\partial^{2} W}{\partial x_{1}^{2}}\left(r e_{1}-\eta y\right) d \sigma(y),
$$

and

$$
\partial_{2} \omega(r, \eta)=\frac{1}{\sigma_{N}} \int_{\partial B(0,1)} \nabla\left(\frac{\partial W}{\partial x_{1}}\right)\left(r e_{1}-\eta y\right) \cdot y d \sigma(y)
$$

away from the diagonal. We need to investigate the behavior of $\omega$ on the diagonal. Let us make the following definition:

Definition 3 (Integrability on hypersurfaces). A radially symmetric function $g \in \mathcal{C}\left(\mathbb{R}^{N} \backslash\{0\}\right)$ is said to be locally integrable on hypersurfaces if

$$
\int_{[0,1]^{N-1}}|g(\hat{x}, 0)| d \hat{x}<+\infty
$$

where $\hat{x}=\left(x_{1}, \ldots, x_{N-1}\right)$, or equivalently, if $\hat{g}(r) r^{N-2}$ is integrable on $(0,1)$ with $g(x)=$ $\hat{g}(|x|)$. By an abuse of notation, we sometimes say $\hat{g}(r)$ is integrable on hypersurfaces.

Lemma 1 (Regularity of the function $\omega)$. Let $W(x)=k(|x|)$ be a radially symmetric potential belonging to $C^{3}\left(\mathbb{R}^{N} \backslash\{0\}\right)$.

(i) If $k^{\prime}(r)$ is locally integrable on hypersurfaces then $\omega \in C\left(\mathbb{R}_{+}^{2}\right)$.

(ii) If $k^{\prime}(r), k^{\prime \prime}(r)$, and $r^{-1} k^{\prime}(r)$ are locally integrable on hypersurfaces then $\omega \in C^{1}\left(\mathbb{R}_{+}^{2}\right)$.

(iii) Suppose $\Delta W$ is negative in a neighborhood of the origin. If $k^{\prime}(r)$ is locally integrable on hypersurfaces but $\Delta W=k^{\prime \prime}+(N-1) r^{-1} k^{\prime}$ is not, then for any $R>0$,

$$
\lim _{\substack{(r, \eta) \notin \mathcal{D} \\(r, \eta) \rightarrow(R, R)}} \partial_{1} \omega(r, \eta)=+\infty .
$$

Before proving the above lemma, let us discuss the result. Obviously the regularity of the function $\omega$ depends only on the behavior of $W$ at the origin. Assume for simplicity that in the neighborhood of the origin, the potential $W$ is a powerlaw, that is $W(x)=k(|x|)=-|x|^{b} / b$ for all $x \in B(0, \varepsilon)$, where $b$ is possibly negative. Note that $k^{\prime}(r)<0$ for $r<\varepsilon$ so the potential 
is repulsive in the short range. Lemma 1 then claims that $\omega$ is continuous if $b>2-N$ and continuously differentiable if $b>3-N$. Statement (iii) says that if $2-N<b \leq 3-N$, then $\omega$ is continuous but its first derivative goes to $+\infty$ as $(r, \eta)$ approaches the diagonal.

Finally, let us remark that(i) is sharp in the sense that the Newtonian potential $|x|^{2-N}$ is the critical one for the integrability on hypersurfaces. Precisely, Newton's Theorem asserts that the function $\omega$ associated to the Newtonian potential is discontinuous, it has a singularity, across the spherical shell. We now prove the Lemma:

Proof. Let us prove (i). The function $\omega(r, \eta)$ can be rewritten as

$$
\omega(r, \eta)=\int_{\partial B(0, \eta)} e_{1} \cdot \nabla W\left(r e_{1}-y\right) \frac{1}{\sigma_{N} \eta^{N-1}} d \sigma(y) .
$$

Seeing $\omega$ as a function of $x=r e_{1}$ and $\eta$, we can apply Lemma 10 from the appendix with $\mathcal{M}_{\eta}:=\partial B(0, \eta), \phi_{\eta}(x):=\left(\sigma_{N} \eta^{N-1}\right)^{-1}$, and $G(x):=e_{1} \cdot \nabla W(x)$. Since $|G(x)|$ is bounded by $\left|k^{\prime}(|x|)\right|$ which is locally integrable on hypersurfaces, we obtain that $\omega \in C\left(\mathbb{R}_{+}^{2}\right)$.

We now turn to the proof of (ii). It is simple to check that

$$
\frac{\partial^{2} W}{\partial x_{i} \partial x_{j}}=k^{\prime \prime}(r) \frac{x_{i} x_{j}}{r^{2}}+k^{\prime}(r) \frac{\delta_{i j}}{r}-k^{\prime}(r) \frac{x_{i} x_{j}}{r^{3}}
$$

and then $\left|\frac{\partial^{2} W}{\partial x_{i} \partial x_{j}}\right|$ is bounded by a radial function which is locally integrable on hypersurfaces given by a linear combination of $k^{\prime \prime}(r)$ and $r^{-1} k^{\prime}(r)$. Moreover, it has the regularity needed in Lemma 10. We now rewrite the derivatives $\partial_{1} \omega(r, \eta)$ and $\partial_{2} \omega(r, \eta)$ in (7) and (8) as

$$
\begin{aligned}
& \partial_{1} \omega(r, \eta)=-\frac{1}{\sigma_{N} \eta^{N-1}} \int_{\partial B(0, \eta)} \frac{\partial^{2} W}{\partial x_{1}^{2}}\left(r e_{1}-y\right) d \sigma(y), \\
& \partial_{2} \omega(r, \eta)=\frac{1}{\sigma_{N} \eta^{N}} \int_{\partial B(0, \eta)} \nabla\left(\frac{\partial W}{\partial x_{1}}\right)\left(r e_{1}-y\right) \cdot y d \sigma(y) .
\end{aligned}
$$

The reader can easily check that Lemma 10 applies similarly as before, so that $\omega \in C^{1}\left(\mathbb{R}_{+}^{2}\right)$.

Finally we prove (iii). Taking the divergence of (4) we obtain:

$$
\left(\operatorname{div} v_{R}\right)(x)=-\Delta W * \delta_{R}(x)=\partial_{1} \omega(|x|, R)+(N-1) \frac{\omega(|x|, R)}{|x|},
$$

and therefore $\partial_{1} \omega(r, \eta)$ can be written:

$$
\partial_{1} \omega(r, \eta)=-\left(\Delta W * \delta_{\eta}\right)\left(r e_{1}\right)-(N-1) \frac{\omega(r, \eta)}{r} .
$$

For $0<\varepsilon<r_{0}$, let $\chi_{\varepsilon} \in C^{\infty}\left(\mathbb{R}_{+}\right)$be a cut-off function, such that $\chi_{\varepsilon}=1$ on $[0, \varepsilon / 2]$, and $\chi_{\varepsilon}=0$ on $[\varepsilon, \infty)$. Choose $\varepsilon$ such that the function $-\Delta W^{\varepsilon}(x):=-\chi_{\varepsilon}(x) \Delta W(x)$ is nonnegative. Using Lemma 11 with $\eta_{1}$ and $\eta_{2}$ such that $\eta_{1}<R<\eta_{2}$, and noting that $\operatorname{dist}\left(r e_{1}, \partial B(0, \eta)\right)=|r-\eta|$, we find that

$$
\lim _{\substack{(r, \eta) \notin \mathcal{D} \\(r, \eta) \rightarrow(R, R)}}-\left(\Delta W^{\varepsilon} * \delta_{\eta}\right)\left(r e_{1}\right)=+\infty .
$$

To conclude the proof, note that the functions $(r, \eta) \mapsto \frac{\omega(r, \eta)}{r}$ and $(r, \eta) \mapsto\left(\left[\left(1-\chi_{\varepsilon}\right) \Delta W\right] *\right.$ $\left.\delta_{\eta}\right)\left(r e_{1}\right)$ are bounded in a neighborhood of $(R, R)$. 
2.3. The two radial instabilities. In this subsection we exhibit some elementary calculations in order to understand under which conditions a spherical shell is a stable steady state. Rigorous results about stability and instability of spherical shell with respect to the transport distance will be provided in section 3 and 4 . This subsection provide motivations for the rigorous results to come later.

Definition 4 (Steady states). A probability measure $\mu \in \mathcal{P}\left(\mathbb{R}^{N}\right)$ is said to be a steady state of the nonlocal interaction equation (1) if

$$
-(\nabla W * \mu)(x)=0 \quad \text { for all } x \in \operatorname{supp}(\mu) .
$$

We now show that if the attractive strength $k^{\prime}(r)$ of a repulsive-attractive potential $W(x)=$ $k(|x|)$ does not decay faster $1 / r^{N}$ as $r \rightarrow \infty$, then there exists a spherical shell steady state.

Lemma 2 (Existence of spherical shell steady states). Let $W(x)=k(|x|)$ be a radially symmetric potential belonging to $C^{1}\left(\mathbb{R}^{N} \backslash\{0\}\right)$ and such that $k^{\prime}(r)$ is locally integrable on hypersurfaces. Let us assume that the potential is repulsive-attractive in the following sense: there exists $R_{a}>0$ such that

$$
k^{\prime}(r) \geq 0 \text { for } r>R_{a}, \quad \text { and } \quad k^{\prime}(r)<0 \text { for } 0<r<R_{a} .
$$

Defining for $r>2 R_{a}$ the function

$$
\Sigma(r):=\inf _{r / 2 \leq s \leq 2 r} k^{\prime}(s) \geq 0,
$$

we will further assume that

$$
\lim _{r \rightarrow \infty} r^{N} \Sigma(r)=+\infty .
$$

Then there exists at least a $R>0$ such that the spherical shell $\delta_{R} \in \mathcal{P}\left(\mathbb{R}^{N}\right)$ is a steady state to (1).

Proof. Note that from (4) we directly obtain that a spherical shell $\delta_{R} \in \mathcal{P}\left(\mathbb{R}^{N}\right)$ is a steady state if and only if $\omega(R, R)=0$, that is, if and only if condition (C0) holds. Since $k^{\prime}(r)$ is locally integrable on hypersurface $\omega \in C\left(\mathbb{R}_{+}^{2}\right)$ due to Lemma 1 . So the function $F(r):=$ $\omega(r, r) \in C\left(\mathbb{R}_{+}\right)$. Using formula (2), we get

$$
F(r)=\frac{1}{\sigma_{N}} \int_{\partial B(0,1)} k^{\prime}\left(r\left|y-e_{1}\right|\right) \frac{y-e_{1}}{\left|y-e_{1}\right|} \cdot e_{1} d \sigma(y) .
$$

Let us remark that $\left(y-e_{1}\right) \cdot e_{1} \leq 0$ for all $y \in \partial B(0,1)$, and thus for $2 r<R_{a}$ we easily get $F(r)>0$. It is enough to show that there exists $r>2 R_{a}$ such that $F(r)<0$. In order to do this, we proceed as in [17, Proposition 2.2] and divide the integral in the definition of $F(r)$ into two sets: $A:=\partial B(0,1) \cap B\left(e_{1}, R_{a} / r\right)$ and its complementary set $A^{c}$. Note that the integrand is positive on $A$ and negative on $A^{c}$. We will show that for $r$ large enough the integral over the set $A^{c}$ is greater in absolute value than the integral over the set $A$. It is easy to see that $B:=\left\{y \in \partial B(0,1)\right.$ such that $\left.2\left|y-e_{1}\right| \geq 1\right\} \subset A^{c}$ and $B \neq \emptyset$ as soon as $r>2 R_{a}$.

We first estimate the integral

$$
\left|\int_{A} k^{\prime}\left(r\left|y-e_{1}\right|\right) \frac{y-e_{1}}{\left|y-e_{1}\right|} \cdot e_{1} d \sigma(y)\right|=\int_{A}\left|k^{\prime}\left(r\left|y-e_{1}\right|\right)\right| \frac{\left(e_{1}-y\right) \cdot e_{1}}{\left|e_{1}-y\right|} d \sigma(y)
$$

Let $\theta$ be the angle between $e_{1}-y$ and $e_{1}$ and note that for all $y \in A:=\partial B(0,1) \cap B\left(e_{1}, R_{a} / r\right)$ we have by the law of cosines

$$
\frac{e_{1}-y}{\left|e_{1}-y\right|} \cdot e_{1}=\cos \theta \leq \frac{R_{a}}{2 r}
$$


Using Lemma 8 from the Appendix with $\mathcal{M}=\partial B(0,1)$ we then obtain

$$
\begin{aligned}
\left|\int_{A} k^{\prime}\left(r\left|y-e_{1}\right|\right) \frac{e_{1}-y}{\left|e_{1}-y\right|} \cdot e_{1} d \sigma(y)\right| & \leq \frac{R_{a}}{2 r} \int_{A}\left|k^{\prime}\left(r\left|y-e_{1}\right|\right)\right| d \sigma(y) \\
& \leq \frac{R_{a}}{2 r} \int_{0}^{R_{a} / r}\left|k^{\prime}(r s)\right|\left|\mathcal{M} \cap\left\{\left|y-e_{1}\right|=s\right\}\right|_{\mathcal{H}^{N-2}} d s \\
& \leq C \frac{R_{a}}{2 r} \int_{0}^{R_{a} / r}\left|k^{\prime}(r s)\right| s^{N-2} d s \\
& =C \frac{R_{a}}{2 r^{N}} \int_{0}^{R_{a}}\left|k^{\prime}(z)\right| z^{N-2} d z \leq \frac{C_{1}}{r^{N}}
\end{aligned}
$$

where we have used the fact that $k^{\prime}(r)$ is integrable on hypersurfaces to obtain the last inequality.

Since the integrand is negative in $A^{c}$ and since $B \subset A^{c}$ for $r>2 R_{a}$ we have:

$$
\int_{A^{c}} k^{\prime}\left(r\left|y-e_{1}\right|\right) \frac{y-e_{1}}{\left|y-e_{1}\right|} \cdot e_{1} d \sigma(y) \leq \int_{B} k^{\prime}\left(r\left|y-e_{1}\right|\right) \frac{y-e_{1}}{\left|y-e_{1}\right|} \cdot e_{1} d \sigma(y) .
$$

Moreover, thanks to the law of cosines, $\frac{y-e_{1}}{\left|y-e_{1}\right|} \cdot e_{1}=\cos (-\theta) \leq-1 / 4$ for $y \in B$, and then using (11)

$$
\begin{aligned}
\int_{B} k^{\prime}\left(r\left|y-e_{1}\right|\right) \frac{y-e_{1}}{\left|y-e_{1}\right|} \cdot e_{1} d \sigma(y) & \leq-\frac{1}{4} \int_{B} k^{\prime}\left(r\left|y-e_{1}\right|\right) d \sigma(y) \\
& \leq-\frac{1}{4} \int_{B} d \sigma(y) \Sigma(r) \leq-C_{2} \Sigma(r) .
\end{aligned}
$$

Condition (11) on $\Sigma(r)$ implies that $C_{2} \Sigma(r) \geq C_{1} / r^{N}$ for $r$ large enough and therefore $F(r)<$ 0 for $r$ large enough. Then the continuity of $F$ implies the existence of a radius $\tilde{r}>0$ such that $F(\tilde{r})=0$.

The following proposition gives some hints about the stability properties of the spherical shell steady states.

Proposition 1 (Instability modes by energy arguments). Assume that the radial interaction potential $W$ is such that $\omega \in C^{1}\left(\mathbb{R}_{+}^{2}\right)$ and let $\delta_{R}$ be a steady state, that is $\omega(R, R)=0$.

(i) If (C1) is not satisfied then by splitting the spherical shell into two spherical shells we can decrease the energy. More precisely there exists $d r_{0}>0$ such that, given $0<|d r|<d r_{0}$,

$$
E\left[(1-\epsilon) \delta_{R}+\epsilon \delta_{R+d r}\right]<E\left[\delta_{R}\right]
$$

if $\epsilon$ is small enough.

(ii) If (C2) is not satisfied then by increasing or decreasing the radius of the spherical shell we can decrease the energy. More precisely there exists $d r_{0}>0$ such that

$$
E\left[\delta_{R+d r}\right]<E\left[\delta_{R}\right]
$$

for all $0<|d r|<d r_{0}$.

Proof. Let us introduce the notations

$$
E[\mu, \nu]:=\frac{1}{2} \iint_{\mathbb{R}^{N} \times \mathbb{R}^{N}} W(x-y) d \mu(x) d \nu(y),
$$


so that $E[\mu, \mu]=E[\mu]$, and

$$
E(r, \eta):=E\left[\delta_{r}, \delta_{\eta}\right]=\frac{1}{2} \frac{1}{\sigma_{N}^{2}} \iint_{\partial B(0,1) \times \partial B(0,1)} W(r x-\eta y) d \sigma(x) d \sigma(y) .
$$

Taking the derivative we get:

$$
\begin{aligned}
\frac{\partial E}{\partial r}(r, \eta) & =\frac{1}{2} \frac{1}{\sigma_{N}^{2}} \iint_{\partial B(0,1) \times \partial B(0,1)} \nabla W(r x-\eta y) \cdot x d \sigma(x) d \sigma(y) \\
& =\frac{1}{2} \frac{1}{\sigma_{N}} \int_{\partial B(0,1)}\left(\frac{1}{\sigma_{N}} \int_{\partial B(0,1)} \nabla W(r x-\eta y) d \sigma(y)\right) \cdot x d \sigma(x) \\
& =\frac{1}{2} \frac{1}{\sigma_{N}} \int_{\partial B(0,1)}\left(\nabla W * \delta_{\eta}(r x)\right) \cdot x d \sigma(x)=-\frac{1}{2} \omega(r, \eta) .
\end{aligned}
$$

Since $E(r, \eta)=E(\eta, r)$, the Hessian matrix of $E(r, \eta)$ is given by

$$
H(r, \eta)=-\frac{1}{2}\left[\begin{array}{ll}
\partial_{1} \omega(r, \eta) & \partial_{2} \omega(r, \eta) \\
\partial_{2} \omega(\eta, r) & \partial_{1} \omega(\eta, r)
\end{array}\right]
$$

If $\delta_{R}$ is a steady state, i.e. $\omega(R, R)=0$, then $\nabla E(R, R)=0$ and

$$
\begin{gathered}
E(R+d r, R)=E(R, R)-\frac{1}{4} \partial_{1} \omega(R, R) d r^{2}+o\left(d r^{2}\right) \\
E(R+d r, R+d r)=E(R, R)-\frac{1}{2}\left(\partial_{1} \omega(R, R)+\partial_{2} \omega(R, R)\right) d r^{2}+o\left(d r^{2}\right)
\end{gathered}
$$

The proof of (ii) follows directly from the Taylor expansion (13). By using the Taylor expansions $(12)$ and $(13)$ as well as the bilinearity of $E[\mu]=E[\mu, \mu]$ :

$$
\begin{gathered}
E\left[(1-\epsilon) \delta_{\partial B(0, R)}+\epsilon \delta_{\partial B(0, R+d r)},(1-\epsilon) \delta_{\partial B(0, R)}+\epsilon \delta_{\partial B(0, R+d r)}\right] \\
=(1-\epsilon)^{2} E(R, R)+2 \epsilon(1-\epsilon) E(R+d r, R)+\epsilon^{2} E(R+d r, R+d r) \\
\left.=E(R, R)-\frac{\epsilon}{2} \partial_{1} \omega(R, R) d r^{2}-\frac{\epsilon^{2}}{2} \partial_{2} \omega(R, R)\right) d r^{2}+o\left(d r^{2}\right)
\end{gathered}
$$

from which (i) follows by taking $\epsilon$ and $d r_{0}$ small enough.

The following elementary Lemma shows that the instability condition $\partial_{1} \omega(R, R)>0$ (i.e. (C1) is not satisfied) simply means that the divergence of the velocity field generated by the spherical shell is positive on the spherical shell. Being the velocity field "expanding", it makes sense that splitting the spherical shell into two reduces the energy as proven in previous Proposition 1 .

Lemma 3 (Divergence of the velocity field). Assume the spherical shell $\delta_{R}$ is a steady state, i.e., condition $(\mathbf{C 0}): \omega(R, R)=0$. Let $v_{R}$ be the velocity field generated by $\delta_{R}$, given by (4). Then

$$
\left(\operatorname{div} v_{R}\right)(x)=\partial_{1} \omega(R, R) \quad \text { for all } x \in \partial B(0, R) .
$$

Proof. This is a direct consequence of 10$)$ together with the fact that $\omega(R, R)=0$. 
2.4. Well-posedness of $L^{p}$-solutions. Global existence and uniqueness of $L^{p}$-solutions of equation (1) was established in [11, Theorem 1] under some conditions on the interaction potential $W$ :

Theorem 1 ( $L^{p}$-Well posedness theory). Consider $1<q<\infty$ and $p$ its Hölder conjugate. Suppose $\nabla W \in \mathcal{W}^{1, q}\left(\mathbb{R}^{N}\right)$ and $\mu_{0} \in L^{p}\left(\mathbb{R}^{N}\right) \cap \mathcal{P}_{2}\left(\mathbb{R}^{N}\right)$ is nonnegative. Then there exists a time $T^{*}>0$ and a nonnegative function $\mu \in C\left(\left[0, T^{*}\right], L^{p}\left(\mathbb{R}^{N}\right)\right) \cap C^{1}\left(\left[0, T^{*}\right], \mathcal{W}^{-1, p}\left(\mathbb{R}^{N}\right)\right)$ such that (1) holds in the sense of distributions in $\mathbb{R}^{N} \times\left(0, T^{*}\right)$ with $\mu(0)=\mu_{0}$. Moreover the second moment of $x \mapsto \mu(t, x)$ remains bounded and the $L^{1}$ norm is conserved. Also, the function $t \rightarrow\|\mu(t)\|_{L^{p}}^{p}$ is differentiable and satisfies

$$
\frac{d}{d t}\left\{\|\mu(t)\|_{L^{p}}^{p}\right\}=-(p-1) \int_{\mathbb{R}^{N}} \mu(t, x)^{p} \operatorname{div} v(t, x) d x \quad \forall t \in\left[0, T^{*}\right] .
$$

Furthermore, if ess sup $\Delta W<+\infty$, then $t \rightarrow\|\mu(t)\|_{L^{p}}^{p}$ does not grow faster than exponentially and we have global well-posedness.

In the above theorem $\mathcal{P}_{2}\left(\mathbb{R}^{N}\right)$ stands for the space of probability measure with finite second moment. We will refer to the solutions provided by the above theorem as $L^{p}$-solutions.

One can find in [4] that the authors extend the global-in-time well posedness $L^{p}$-theory to repulsive-attractive potentials under suitable conditions. We summarize the result in the following theorem.

Theorem 2 (Dealing with possibly growing at $\infty$ attractive potentials). Assume that $W(x)=$ $k(|x|)$ is a radially symmetric repulsive-attractive potential, $W(x)=\left(W_{R}+W_{A}\right)(x)=\left(k_{R}+\right.$ $\left.k_{A}\right)(|x|)=k(|x|)$ with $k \in C^{2}((0,+\infty)), W_{A}$ attractive (i.e. $\left.k_{A}^{\prime}>0\right)$, with $\nabla W \in \mathcal{W}_{\text {loc }}^{1, q}\left(\mathbb{R}^{N}\right)$, $1<q<\infty$, and $W_{R}$ compactly supported repulsive $\left(k_{R}^{\prime} \leq 0\right)$. Furthermore, assume that $k$ satisfies:

(i) $\exists \delta_{1}>0$ such that $k^{\prime \prime}(r)$ is monotonic in $\left(0, \delta_{1}\right)$.

(ii) $\exists \delta_{2}>0$ such that $r k^{\prime \prime}(r)$ is monotonic in $\left(0, \delta_{2}\right)$.

(iii) $D:=\sup _{r \in(0, \infty)}\left|k_{R}^{\prime}(r)\right|<\infty$

(iv) There exists $m$ such that $\frac{k_{A}(r)}{1+r^{m}}$ is bounded and increasing.

Then there exists a global in time solution for the equation (1) with compactly supported initial data $\mu_{0} \in L^{p}\left(\mathbb{R}^{N}\right)$, which is compactly supported for all $t \geq 0$.

\section{The Fattening instability and Dimensionality of the Steady STATE}

3.1. The radially symmetric case. This first subsection concerns radially symmetric solutions. We show that if the singularity of $W$ at the origin is such that the kernel $\omega$ is $C^{1}$, and if condition (C1) is not satisfied, then a radially symmetric solution can not converge weakly toward the spherical shell stationary state. We also show that the same result holds if the singularity of $W$ at the origin is so strong that the kernel $\omega$ is not $C^{1}$ (and this is independent of how strong the attractive part of the potential is).

Theorem 3 (Instability of spherical shells: radially symmetric case). Let $W(x)=k(|x|)$ be a radially symmetric potential belonging to $C^{3}\left(\mathbb{R}^{N} \backslash\{0\}\right)$ and such that $k^{\prime}(r)$ is locally integrable on hypersurface (so that $\omega$ is continuous). Assume that the spherical shell $\delta_{R}$ is a steady state, that is, (C0): $\omega(R, R)=0$, and that one of the two following hypotheses hold:

(i) $k^{\prime \prime}(r)$ and $r^{-1} k^{\prime}(r)$ are locally integrable on hypersurfaces (so that $\omega$ is $C^{1}$ ), and $\partial_{1} \omega(R, R)>0$. 
(ii) $\Delta W$ is negative in a neighborhood of the origin and is not locally integrable on hypersurfaces (in which case $\omega$ is not $C^{1}$ and $\left.\lim _{\substack{(r, \eta) \rightarrow(R, \cap) \\(r, \eta)}} \partial_{1} \omega(r, \eta)=+\infty\right)$.

Then it is not possible for an $L^{p}$ radially symmetric solution of (5)-(6) to converge weakly-* as measures to $\delta_{R}$ as $t \rightarrow \infty$.

To clarify the result, let us consider the case where the repulsive-attractive potential $W(x)=k(|x|)$ has its repulsive part described by a powerlaw. Let say, for example, that $k(r)=-r^{b} / b$, for all $r<1$ and $k^{\prime}(r)>0$ for $r>2$. If $2-N<b \leq 3-N$, then $\Delta W$ is not locally integrable on hypersurfaces and therefore, according to (ii), whatever is the behavior of $W(x)$ for $|x|>1, L^{p}$ radially symmetric solutions can not converge toward the steady state. In other words if the repulsive singularity of the potential is equal to or stronger than $|x|^{3-N}$ then the potential is so repulsive in the short range that solution can not concentrate on a spherical shell, and this is independent of how attractive the potential is in the long range. On the other hand if $b>3-N$ then the kernel $\omega$ is $C^{1}$. In this case, the balance between the repulsive part and the attractive part of the potential dictates whether or not the spherical shell is an attractor: if $\partial_{1} \omega(R, R)>0$, then the repulsive part dominates and the spherical shell is not an attractor.

We remind, see [59, 19, 45], that for $1 \leq p<\infty$ the distance $d_{p}$ between two measures $\nu, \rho$ is defined by

$$
d_{p}^{p}(\nu, \rho)=\inf _{\pi \in \Pi(\nu, \rho)}\left\{\int_{\mathbb{R}^{N} \times \mathbb{R}^{N}}|x-y|^{p} d \pi(x, y)\right\},
$$

where $\Pi(\nu, \rho)$ is the set of those joint distribution functions with marginals $\nu$ and $\rho$. When $p=+\infty$ then the distance is defined as

$$
d_{\infty}(\nu, \rho)=\inf _{\mathcal{T}: \mathbb{R}^{N} \longrightarrow \mathbb{R}^{N}}\left\{\sup _{y \in \mathbb{R}^{N}}|y-\mathcal{T}(y)|: \mathcal{T} \# \rho=\nu\right\} .
$$

We now prove the Theorem.

Proof. If conditions (ii) of the Theorem holds, then from (9) of Lemma 1, it is clear that there exists $\delta>0$ such that

$$
\forall \eta \in(R-\delta, R+\delta), r \rightarrow \omega(r, \eta) \text { is strictly increasing in }(R-\delta, R+\delta) .
$$

Of course (16) also trivially holds if condition (i) of the Theorem is satisfied. We proceed by contradiction. Assume that $\mu(x, t)=\mu_{t}(x)$ is an $L^{p}$ radially symmetric solution which converges weakly-* as measures to a spherical shell of radius $R$ as $t \rightarrow \infty$.

Step 1. Assume first that $\mu_{t}$ converges toward $\delta_{R}$ not only weakly-* as measures but also with respect to the $d_{\infty}$-topology. This implies that the support of the radial solution $\hat{\mu}_{t}$ to (5) converge to the point $\{R\}$. Choose $T>0$ such that $\operatorname{supp}\left(\hat{\mu}_{t}\right) \subset(R-\delta, R+\delta)$ for all $t>T$. Using the monotonicity property (16) we obtain that for $t \geq T$ and for $R-\delta<r_{1}<r_{2}<R+\delta$

$$
\hat{v}\left(t, r_{2}\right)-\hat{v}\left(t, r_{1}\right)=\int_{R-\delta}^{R+\delta} \omega\left(r_{2}, \eta\right)-\omega\left(r_{1}, \eta\right) d \hat{\mu}_{t}(\eta) \geq 0
$$

where $\hat{v}$ is the velocity field in radial coordinate defined by (6). Therefore for all $t \geq T$ the function $r \rightarrow \hat{v}(t, r)$ is increasing on $(R-\delta, R+\delta)$. Let $r_{1}(t)$ and $r_{2}(t)$ be two solutions of the ODE $r_{i}^{\prime}(t)=\hat{v}\left(t, r_{i}(t)\right), i=1,2$. Since

$$
\frac{d}{d t}\left(r_{2}(t)-r_{1}(t)\right)^{2}=2\left(r_{2}(t)-r_{1}(t)\right)\left(\hat{v}\left(t, r_{2}(t)\right)-\hat{v}\left(t, r_{1}(t)\right)\right) \geq 0,
$$


we easily see that if for some time $t \geq T, r_{1}(t)$ and $r_{2}(t)$ are in $(R-\delta, R+\delta)$, then their distance increases. This contradicts the fact that the support of $\hat{\mu}_{t}$ is converging to the point $\{R\}$ as $t \rightarrow \infty$. Let us be more precise. Since $\mu_{T}$ is supported in $(R-\delta, R+\delta)$ and is absolutely continuous with respect to the Lebesgue measure, there exists $R_{1}$ and $R_{2}$ in $(R-\delta, R+\delta), R_{1} \neq R_{2}$, such that $\int_{0}^{R_{1}} \hat{\mu}_{T}(x) d x=1 / 3$ and $\int_{R_{2}}^{\infty} \hat{\mu}_{T}(x) d x=1 / 3$. Consider the ODEs $r_{i}^{\prime}(t)=\hat{v}\left(t, r_{i}(t)\right), r_{i}(T)=R_{i}, i=1,2$. Clearly $r_{1}(t)$ and $r_{2}(t)$ remain in $\left(R-\delta, R+\delta\right.$ ) for all $t \geq T$ (otherwise the support of $\mu_{t}$ would not stay in $(R-\delta, R+\delta)$ ). So $\left|r_{2}(t)-r_{1}(t)\right| \geq\left|R_{2}-R_{1}\right|$ for all $t \geq T$ and the support of $\mu_{t}$ can not converge to the point $\{R\}$, which contradicts our assumption.

Step 2. Assume now that $\mu_{t}$ converges weakly toward $\delta_{R}$ but does not converge with respect to the $d_{\infty}$-topology. From the continuity of the function $\eta \rightarrow \omega(r, \eta)$ together with (6) it is clear that $\hat{v}(r, t)$ converges pointwise to $\omega(r, R)$ as $t \rightarrow \infty$. Since the support of $\hat{\mu}_{t}$ does not converge to the set $\{R\}$ there is a sequence of times at which there is always non-zero mass in $(0, R-\epsilon) \cup(R+\epsilon,+\infty)$. Since $\omega(R, R)=0$, the monotonicity condition (16) implies that $\omega(r, R)<0$ for all $r \in(R-\epsilon, R)$ and $\omega(r, R)>0$ for all $r \in(R, R+\epsilon)$ as long as $\epsilon<\delta$. Because of the pointwise convergence of $\hat{v}$ there exists a time $T>0$ such that for all $t>T, \hat{v}(t, R-\epsilon)<0$ and $\hat{v}(t, R+\epsilon)>0$. So after this time $T$ mass cannot enter the region $[R-\epsilon, R+\epsilon]$. This together with the existence of a time $t>T$ for which there is some mass in the complementary of $[R-\epsilon, R+\epsilon]$ contradict the weak convergence towards $\delta_{R}$.

Remark 1. In Step 2 of this proof, since we are dealing with radially symmetric solutions, the problem is essentially one dimensional and the characteristics are ordered. This allows us to exclude the possibility of an $L^{p}$ solution converging toward a spherical shell even if this convergence is very weak and the support of the solution does not converge. In the non radially symmetric case we will be only able to exclude convergence in $d_{\infty}$.

3.2. The non-radially symmetric case. In this subsection we consider non-radially symmetric solutions and we investigate whether it is possible for an $L^{p}$-solution to converge toward a steady state supported on an hypersurface which not necessarily a sphere. Indeed in numerical simulations [39, 60], it is observed that depending on the choice of the repulsive-attractive potential $W$, solutions of (1) can either converge to steady states which are smooth densities or to singular steady states which are measures supported on an hypersurface. We consider steady states $\bar{\mu}$ of the form

$$
\int_{\mathbb{R}^{N}} f(x) d \bar{\mu}(x)=\int_{\mathcal{M}} f(x) \phi(x) d \sigma(x) \quad \forall f \in C\left(\mathbb{R}^{N}\right)
$$

where $\mathcal{M}$ is a compact $C^{2}$ hypersurface and $d \sigma$ is the volume element on $\mathcal{M}$. Roughly speaking, we prove that if the the potential is as singular or more singular than $|x|^{3-N}$ at the origin, then it is not possible for an $L^{p}$-solution to converge toward such a steady state with respect to the $d_{\infty}$-topology. We also prove that the same result holds if the potential is less singular than $|x|^{3-N}$, and if the divergence of the velocity field generated by such steady state is strictly positive on its support.

Theorem 4 (Instability of Spherical Shells: Nonradial case). Let $W(x)=k(|x|)$ be a radially symmetric potential which belongs to $C^{2}\left(\mathbb{R}^{N} \backslash\{0\}\right)$. Assume that $\lim _{r \rightarrow 0} \widehat{\Delta W}(r)=-\infty$ and that close to the origin $\widehat{\Delta W}(r)$ is monotone. Let $\bar{\mu}$ be a steady state of the form (17) with $\mathcal{M}$ being a compact $C^{2}$ hypersurface and let $\bar{v}$ be the velocity field generated by $\bar{\mu}$, that is $\bar{v}=-\nabla W * \bar{\mu}$. If one of the two condition holds: 
(i) $\Delta W$ is locally integrable on hypersurfaces, $\phi \in L^{\infty}(\mathcal{M})$ and

$$
(\operatorname{div} \bar{v})(x):=-(\Delta W * \bar{\mu})(x)>0 \quad \text { for all } x \in \operatorname{supp} \bar{\mu}
$$

(ii) $\Delta W$ is not locally integrable on hypersurfaces and $\phi(x) \geq \phi_{0}>0$ for all $x \in \mathcal{M}$, then it is not possible for an $L^{p}$ solution of (1) to converge to $\bar{\mu}$ with respect to the $d_{\infty}$-topology as $t \rightarrow \infty$.

Before to prove this Theorem, let us make some remarks:

Remark 2. According to Lemma 3, the result of Theorem 3 (i) of the previous subsection can be reformulated as follows: assume that the spherical shell $\delta_{R}$ is a steady state and let $v_{R}$ be its velocity field. If

$$
\left(\operatorname{div} v_{R}\right)(x)=-\left(\Delta W * \delta_{R}\right)(x)>0 \quad \text { for all } x \in \partial B(0, R)
$$

then it is not possible for an $L^{p}$ radially symmetric solution to converge weakly-* as measures to $\delta_{R}$ as $t \rightarrow \infty$. So conditions (i) of Theorems 3 and 4 are essentially the same. Similarly condition (ii) of both Theorems are also essentially the same. In this sense Theorem 4 can be seen as a generalization of Theorem 3 to the non-radially symmetric case.

Remark 3. The assumption $\lim _{r \rightarrow 0} \widehat{\Delta W}(r)=-\infty$ simply guarantees that the potential $W$ is strongly repulsive at the origin. The monotonicity of $\widehat{\Delta W}(r)$ in a neighborhood of the origin is not essential to the proof and could be replaced by weaker hypotheses. But in practice all potentials of interest satisfy this monotonicity condition.

Remark 4. Part (ii) of the Theorem, roughly speaking, states that if the repulsive-attractive potential $W$ is more singular than $|x|^{3-N}$ at the origin, then whatever is its attractive part, it is not possible for an $L^{p}$ solution of (1) to converge with respect to the $d_{\infty}$-topology toward a singular steady state supported on an hypersurface. So we see that the dimensionality of stable steady states depends on the degree of singularity of the potential. For such potential with a strong repulsive singularity at the origin, steady states are expected to be absolutely continuous with respect to the Lebesgue measure.

Theorem 4 is a direct consequence of the three Lemmas to follow.

Lemma 4 (Approximating the divergence of the velocity field). Let $W$ be as stated in Theorem 4 and let $\bar{\mu}$ be a compactly supported probability measure not belonging to $L^{p}$. Suppose there exists a Hölder continuous function $\widehat{\Delta W} \geq \Delta W$ such that

$$
-\widetilde{\Delta W} * \bar{\mu}>0 \quad \text { on } \operatorname{supp}(\bar{\mu}),
$$

then it is not possible for an $L^{p}$ solution of (1) to converge to $\bar{\mu}$ with respect to the $d_{\infty}$-topology as $t \rightarrow \infty$.

Proof. We proceed by contradiction. Let $\mu_{t}$ be an $L^{p}$ solution such that $\lim _{t \rightarrow \infty} d_{\infty}\left(\mu_{t}, \bar{\mu}\right)=0$. We are going to show that there exists a $T>0$ and an $\epsilon>0$ such that for all $t>T$

$$
\left(\Delta W * \mu_{t}\right)(x)<-\epsilon \quad \text { for all } x \in \operatorname{supp}\left(\mu_{t}\right),
$$

and combined with the equality $(15)$ :

$$
\frac{d}{d t}\left\|\mu_{t}\right\|_{L^{p}}^{p}=(p-1) \int_{\mathbb{R}^{N}}\left(\Delta W * \mu_{t}\right) \mu_{t}^{p} d x,
$$


this guarantees that a subsequence $\mu_{t_{n}}$ converges weakly in $L^{p}$ to an $L^{p}$ function, which contradicts the assumption that $\bar{\mu} \notin L^{p}$. Let us prove $(20)$. Write

$$
\Delta W * \mu_{t}=\widetilde{\Delta W} *\left(\mu_{t}-\bar{\mu}\right)+\widetilde{\Delta W} * \bar{\mu}+(\Delta W-\widetilde{\Delta W}) * \mu_{t}
$$

and note that since $\widetilde{\Delta W} \geq \Delta W$ the third term is negative for all $t$ and $x$. Since $\widetilde{\Delta W}$ is continuous so is $\widetilde{\Delta W} * \bar{\mu}$ and, therefore, $\sqrt{19}$ implies that there exists an $\epsilon>0$ and an open set $\Omega$ containing the support of $\bar{\mu}$ such that $\widetilde{\Delta W} * \bar{\mu}<-\frac{\epsilon}{2}$ on $\Omega$. Here we used that the $\operatorname{supp}(\bar{\mu})$ is a compact manifold. Note that since $\lim _{t \rightarrow \infty} d_{\infty}\left(\mu_{t}, \bar{\mu}\right)=0$ the support of $\mu_{t}$ will eventually be in $\Omega$. To estimate the first term of $(21)$, we consider $\mathcal{T}_{t}: \mathbb{R}^{N} \rightarrow \mathbb{R}^{N}$ a map pushing forward $\mu_{t}$ to $\mu$, i.e. $\mathcal{T}_{t} \# \mu_{t}=\bar{\mu}$. Then

$$
\begin{aligned}
\left\|\widetilde{\Delta W} *\left(\mu_{t}-\bar{\mu}\right)(x)\right\|_{L^{\infty}\left(\mathbb{R}^{N}\right)} & \leq \int_{\mathbb{R}^{N}}\left|\widetilde{\Delta W}(x-y)-\widetilde{\Delta W}\left(x-\mathcal{T}_{t}(y)\right)\right| d \mu_{t}(y) \\
& \left.\leq \int_{\mathbb{R}^{N}} c \mid y-\mathcal{T}_{t}(y)\right)\left.\right|^{\beta} d \mu_{t}(y)
\end{aligned}
$$

since this inequality is true for any map $\mathcal{T}_{t}$ pushing forward $\mu_{t}$ to $\mu$,

$$
\left\|\widetilde{\Delta W} *\left(\mu_{t}-\bar{\mu}\right)\right\|_{L^{\infty}\left(\mathbb{R}^{N}\right)} \leq c d_{\infty}\left(\mu_{t}, \bar{\mu}\right)^{\beta}
$$

so that for $t \geq T$ with $T$ large enough,

$$
\left\|\widetilde{\Delta W} *\left(\mu_{t}-\bar{\mu}\right)\right\|_{L^{\infty}\left(\mathbb{R}^{N}\right)} \leq \frac{\epsilon}{4}
$$

Since $\widetilde{\Delta W} \geq \Delta W$, the last term of $(21)$ is negative, so that $\Delta W * \mu_{t}<0$.

In order to conclude the proof we now need to show that under the hypotheses of the theorem there exists a Hölder continuous function $\widetilde{\Delta W} \geq \Delta W$ satisfying (19). Define

$$
\Delta W^{\epsilon}(x):=\left\{\begin{array}{ll}
\Delta W(x) & \text { if }|x| \geq \epsilon \\
\Delta W\left(\epsilon e_{1}\right) & \text { if }|x|<\epsilon
\end{array} .\right.
$$

The function $\Delta W^{\epsilon}(x)$ is obviously Hölder continuous and, due to the monotonicity of $\Delta W$ around the origin we have $\Delta W^{\epsilon} \geq \Delta W$ for $\epsilon$ small enough. We are left to show that $\Delta W^{\epsilon} * \bar{\mu}<$ 0 on the support of $\bar{\mu}$ for $\epsilon$ small enough and this is done in the following two Lemmas.

Lemma 5 (Continuity in $\epsilon$ of the divergence of the velocity field). Let $\bar{\mu}, \mathcal{M}$ and $W$ be as stated in Theorem 4 (i). Then $\Delta W^{\epsilon} * \bar{\mu}$ converges uniformly on $\mathcal{M}$ toward $\Delta W * \bar{\mu}$. Therefore there exists $\epsilon>0$ such that $\Delta W^{\epsilon} * \bar{\mu}<0$ on the support of $\bar{\mu}$.

Proof. Since $\mathcal{M}$ is $C^{2}$ and compact, using Lemma 8 from the appendix, there exist constants $\delta, C_{1}, C_{2}>0$ so that

$$
C_{1} \int_{0}^{\epsilon} g(r) r^{N-2} d r \leq \int_{\mathcal{M} \cap B(x, \epsilon)} g(|x-y|) d \sigma(y) \leq C_{2} \int_{0}^{\epsilon} g(r) r^{N-2} d r
$$

for all $x \in \mathcal{M}$, for all $\epsilon<\delta$ and for all nonnegative function $g$ locally integrable on hypersurfaces. Since $\Delta W(x)$ is radial and goes to $-\infty$ monotonically as $|x| \rightarrow 0^{+}$, we clearly have that $\Delta W\left(\epsilon e_{1}\right)-\Delta W(x-y) \geq 0$ for all $y \in B(x, \epsilon)$ if $\epsilon$ is small enough. Then we obtain that, 
for all $x \in \mathcal{M}$,

$$
\begin{aligned}
\left|\left(\Delta W^{\epsilon} * \bar{\mu}\right)(x)-(\Delta W * \bar{\mu})(x)\right| & =\int_{\mathcal{M} \cap B(x, \epsilon)}\left[\Delta W\left(\epsilon e_{1}\right)-\Delta W(x-y)\right] \phi(y) d \sigma(y) \\
& \leq C_{2}\|\phi\|_{L^{\infty}(\mathcal{M})} \int_{0}^{\epsilon}[\widehat{\Delta W}(\epsilon)-\widehat{\Delta W}(r)] r^{N-2} d r \\
& \leq C_{2}\|\phi\|_{L^{\infty}(\mathcal{M})} \int_{0}^{\epsilon}[-\widehat{\Delta W}(r)] r^{N-2} d r
\end{aligned}
$$

and we conclude using the fact that $\Delta W$ is integrable on hypersurfaces.

Lemma 6. Let $\bar{\mu}, \mathcal{M}$ and $W$ be as stated in Theorem 4 (ii). Then there exists $\epsilon>0$ such that $\Delta W^{\epsilon} * \bar{\mu}<0$ on the support of $\bar{\mu}$.

Proof. Choose $r_{0}$ as in Lemma 8 and also small enough so that $\widehat{\Delta W}(r) \leq 0$ for all $r \leq r_{0}$. For $\epsilon<r_{0}$ we then have

$$
\left(\Delta W^{\epsilon} * \bar{\mu}\right)(x) \leq \int_{\epsilon \leq|x-y| \leq r_{0}} \Delta W(x-y) d \bar{\mu}(y)+\int_{|x-y|>r_{0}} \Delta W(x-y) d \bar{\mu}(y) .
$$

Since $\Delta W$ is bounded on $B(0,2 \operatorname{diam}(\mathcal{M})) \backslash B\left(0, r_{0}\right)$, the second term is uniformly bounded for $x \in \mathcal{M}$. We use Lemma 8 to estimate the first term of $(22)$ :

$$
\begin{aligned}
& \int_{\epsilon \leq|x-y| \leq r_{0}} \Delta W(x-y) d \bar{\mu}(y)=\int_{\mathcal{M} \cap\left\{y: \epsilon \leq|x-y| \leq r_{0}\right\}} \widehat{\Delta W}(|x-y|) \phi(y) d \sigma(y) \\
& \leq \phi_{0} \int_{\epsilon}^{r_{0}} \widehat{\Delta W}(r)|\mathcal{M} \cap \partial B(x, r)|_{\mathcal{H}^{N-2}} d r \leq \tilde{C} \phi_{0} \int_{\epsilon}^{r_{0}} \widehat{\Delta W}(r) r^{N-2} d r,
\end{aligned}
$$

and since $\Delta W$ is not locally integrable on hypersurface and $\widehat{\Delta W}<0$ on $\left[0, r_{0}\right]$, the last integral goes to $-\infty$ as $\epsilon \rightarrow 0$. Then, for $\varepsilon>0$ small enough, $\Delta W^{\epsilon} * \bar{\mu}<0$ on $\operatorname{supp}(\bar{\mu})$.

\section{Stability for Radial Perturbations}

In this section, we give sufficient conditions for the stability under radial perturbations of $\delta_{R}$ stationary solutions in transport distances for the system (5)-(6). Let us denote by $\mathcal{P}_{2}^{r}\left(\mathbb{R}^{N}\right)$ the set of radial probability measures with bounded second moment.

Here, we will work with radial solutions with the following hypotheses of minimal regularity (HMR): we assume that for any given $\mu_{0} \in \mathcal{P}_{2}^{r}\left(\mathbb{R}^{N}\right)$, there exists $\mu \in A C\left([0, T], \mathcal{P}_{2}^{r}\left(\mathbb{R}^{N}\right)\right)$, with $\mu_{t}=\mu_{0}$ for $t=0$, such that

$$
\left.\hat{v}(t, r)=\int_{0}^{+\infty} \omega(r, \eta) d \hat{\mu}_{t}(\eta) \in L^{2}\left((0, T) \times \mathbb{R}^{N}\right)\right)
$$

for all $T>0$ and their corresponding radial measures $\hat{\mu}_{t}$ satisfy (5) in the weak distributional sense. Moreover, they satisfy that $\int_{0}^{\infty} r d \hat{\mu}_{t}(r)$ is an absolutely continuous function in time for which

$$
\frac{d}{d t} \int_{0}^{\infty} r d \hat{\mu}_{t}(r)=\int_{0}^{\infty} \hat{v}(t, r) d \hat{\mu}_{t}(r)
$$

holds a.e. $t \geq 0$. Furthermore, if $\hat{\mu}_{0}$ is compactly supported, we assume that

$$
r_{1}(t)=\min \left\{\operatorname{supp}\left(\hat{\mu}_{t}\right)\right\} \quad \text { and } \quad r_{2}(t)=\max \left\{\operatorname{supp}\left(\hat{\mu}_{t}\right)\right\}
$$

are absolutely continuous functions with $\frac{d}{d t} r_{i}(t)=\hat{v}\left(t, r_{i}(t)\right)$ a.e. $t \geq 0, i=1,2$. 
The existence theory developed in Section 5 ensures that smooth classical solutions satisfying (HMR) exist for $\mu_{0} \in \mathcal{P}_{2}^{r}\left(\mathbb{R}^{N}\right) \cap \mathcal{W}^{2, \infty}\left(\mathbb{R}^{N}\right)$ initial data under suitable assumptions on the potential. Therefore, we assume in this section that our radial solutions satisfy (5)-(6) with $\omega$ given by (2) verifying suitable hypotheses specified in each result.

Theorem 5 (Stability for local perturbations). Assume $\omega \in C^{1}\left(\mathbb{R}_{+}^{2}\right)$ as given in (2) and that $\delta_{R}$ is a stationary solution to (5)-(6), that is, the condition $(\mathbf{C 0}): \omega(R, R)=0$. Let us assume that $(\mathbf{C 1})$ and $(\mathbf{C 2})$ are satisfied with strict inequality, that is:

$$
\partial_{1} \omega(R, R)<0 \quad \text { and } \quad \partial_{1} \omega(R, R)+\partial_{2} \omega(R, R)<0 .
$$

Then there exists $\varepsilon_{0}>0$ such that if the initial data $\mu_{0} \in \mathcal{P}_{2}^{r}\left(\mathbb{R}^{N}\right)$ satisfies $\operatorname{supp}\left(\hat{\mu}_{0}\right) \subset$ $\left[R-\varepsilon_{0}, R+\varepsilon_{0}\right]$, and for any solution to (5)-(6) with initial data satisfying (HMR) we get

$$
d_{2}\left(\hat{\mu}_{t}, \delta_{R}\right) \leq C e^{-\gamma t}
$$

for any $0<\gamma<-\max \left(\partial_{1} \omega(R, R), \frac{d}{d R} \omega(R, R)\right)$ for suitable $C$.

Proof of the Theorem. Since we have assumed that the solutions to (5)-(6) satisfy the regularity conditions $(\mathbf{H M R})$, then $\Gamma(t):=\operatorname{diam}\left(\operatorname{supp}\left(\hat{\mu}_{t}\right)\right)=r_{2}(t)-r_{1}(t)$, and

$$
\Theta(t):=\int_{0}^{\infty} r d \hat{\mu}_{t}(r)-R
$$

are absolutely continuous function of $t \geq 0$. We will proceed by contradiction.

We define $T:=\min \left\{t \geq 0 ; \Gamma(t)+|\Theta(t)| \geq 4 \varepsilon_{0}\right\}$ and let us assume that $T<\infty$ for all $\varepsilon_{0}>0$ close to 0 . Note that $T>0$ by continuity of $\Gamma(t)+|\Theta(t)|$, since supp $\left(\hat{\mu}_{0}\right) \subset\left[R-\varepsilon_{0}, R+\varepsilon_{0}\right]$ implies that

$$
\Gamma(0)+|\Theta(0)| \leq 2 \varepsilon_{0}+\int_{R-\varepsilon_{0}}^{R+\varepsilon_{0}}|r-R| d \hat{\mu}_{0}(r) \leq 3 \varepsilon_{0} .
$$

Now, for $t \in[0, T], \operatorname{supp}\left(\hat{\mu}_{t}\right) \subset\left[R-4 \varepsilon_{0}, R+4 \varepsilon_{0}\right]$, since

$$
\begin{aligned}
\left|r_{i}(t)-R\right| & \leq\left|r_{i}(t)-(R+\Theta(t))\right|+|\Theta(t)| \leq\left(r_{2}(t)-r_{1}(t)\right)+|\Theta(t)| \\
& =\Gamma(t)+|\Theta(t)| \leq 4 \varepsilon_{0},
\end{aligned}
$$

using that the center of mass $\Theta(t)+R$ is obviously in $\left[r_{1}(t), r_{2}(t)\right]$, for all $t \geq 0$ and the definition of $T$.

Then, for $t \in[0, T]$, Taylor expanding to order one and using that $\partial_{1} \omega$ is uniformly continuous on $\left[R-4 \varepsilon_{0}, R+4 \varepsilon_{0}\right]^{2}$ together with (HMR), we get

$$
\begin{aligned}
\frac{d}{d t} \Gamma(t) & =\frac{d}{d t} r_{2}(t)-\frac{d}{d t} r_{1}(t)=\hat{v}\left(t, r_{2}(t)\right)-\hat{v}\left(t, r_{1}(t)\right)=\int_{0}^{\infty}\left[\omega\left(r_{2}(t), \eta\right)-\omega\left(r_{1}(t), \eta\right)\right] d \hat{\mu}_{t}(\eta) \\
& =\int_{0}^{\infty}\left[\partial_{1} \omega\left(r_{1}(t), \eta\right)\left(r_{2}(t)-r_{1}(t)\right)+g\left(r_{1}(t), r_{2}(t), \eta\right)\right] d \hat{\mu}_{t}(\eta),
\end{aligned}
$$

where $g$ satisfies

$$
\lim _{\left|r_{2}-r_{1}\right| \rightarrow 0}\left(\sup _{\eta \in\left[r_{1}, r_{2}\right]} \frac{\left|g\left(r_{1}, r_{2}, \eta\right)\right|}{\left|r_{2}-r_{1}\right|}\right)=0
$$

Since (26) is satisfied, the integral of $g$ can be estimated as follows

$$
\int_{0}^{\infty} g\left(r_{1}(t), r_{2}(t), \eta\right) d \hat{\mu}_{t}(\eta)=\int_{r_{1}(t)}^{r_{2}(t)} g\left(r_{1}(t), r_{2}(t), \eta\right) d \hat{\mu}_{t}(\eta)=o\left(r_{2}(t)-r_{1}(t)\right)=o(\Gamma(t)) .
$$


Proceeding with the same argument as before using (26), we can estimate

$$
\begin{aligned}
\frac{d}{d t} \Gamma(t) & =\left(r_{2}(t)-r_{1}(t)\right) \int_{0}^{\infty} \partial_{1} \omega\left(r_{1}(t), \eta\right) d \hat{\mu}_{t}(\eta)+o(\Gamma) \\
& =\left(r_{2}(t)-r_{1}(t)\right) \int_{0}^{\infty}\left[\partial_{1} \omega(R, R)+\left(\partial_{1} \omega\left(r_{1}(t), \eta\right)-\partial_{1} \omega(R, R)\right)\right] d \hat{\mu}_{t}(\eta)+o(\Gamma) .
\end{aligned}
$$

Since $\eta \in \operatorname{supp}\left(\hat{\mu}_{t}\right)=\left[r_{1}(t), r_{2}(t)\right] \subset\left[R-4 \varepsilon_{0}, R+4 \varepsilon_{0}\right]$ thanks to (25), we can then use the uniform continuity of $\partial_{1} \omega$ on $\left[R-4 \varepsilon_{0}, R+4 \varepsilon_{0}\right]^{2}$ to get:

$$
\left|\partial_{1} \omega\left(r_{1}(t), \eta\right)-\partial_{1} \omega(R, R)\right| \leq C\left|r_{1}(t)-R\right|+|\eta-R| \leq C\left|r_{1}(t)-R\right|+\left|r_{2}(t)-R\right|,
$$

for any $\eta \in \operatorname{supp}\left(\hat{\mu}_{t}\right)$. We can then use 25) again giving

$$
\frac{d}{d t} \Gamma(t)=\partial_{1} \omega(R, R)\left(r_{2}(t)-r_{1}(t)\right)+o(\Gamma)+o(|\Theta|)
$$

On the other hand, we can also estimate using (23)

$$
\begin{aligned}
\frac{d}{d t} \Theta(t) & =\int_{0}^{\infty} \hat{v}(r, t) d \hat{\mu}_{t}(r)=\int_{0}^{\infty} \int_{0}^{\infty} \omega(r, \eta) d \hat{\mu}_{t}(r) d \hat{\mu}_{t}(\eta) \\
& =\int_{0}^{\infty} \int_{0}^{\infty}\left[\omega(\eta, \eta)+\partial_{1} \omega(\eta, \eta)(r-\eta)\right] d \hat{\mu}_{t}(r) d \hat{\mu}_{t}(\eta)+o(\Gamma)+o(|\Theta|),
\end{aligned}
$$

where we have again used an argument as in 26 to estimate the rest term of the Taylor expansion, and we use it once again to obtain

$$
\begin{aligned}
\frac{d}{d t} \Theta(t)= & \int_{0}^{\infty} \int_{0}^{\infty}\left[\omega(R, R)+\frac{d}{d R} \omega(R, R)(\eta-R)+\partial_{1} \omega(\eta, \eta)(r-\eta)\right] d \hat{\mu}_{t}(r) d \hat{\mu}_{t}(\eta) \\
& +o(\Gamma)+o(|\Theta|) \\
= & \frac{d}{d R} \omega(R, R)\left(\int_{0}^{\infty} \eta d \hat{\mu}_{t}(\eta)-R\right)+\partial_{1} \omega(R, R)\left(\int_{0}^{\infty} r d \hat{\mu}_{t}(r)-\int_{0}^{\infty} \eta d \hat{\mu}_{t}(\eta)\right) \\
& +o(\Gamma)+o(|\Theta|) \\
= & \left(\frac{d}{d R} \omega(R, R)\right) \Theta+o(\Gamma)+o(|\Theta|) .
\end{aligned}
$$

We now combine the estimates on $\Gamma$ and $\Theta$ to get:

$$
\frac{d}{d t}(\Gamma+|\Theta|)(t) \leq \max \left(\partial_{1} \omega(R, R), \frac{d}{d R} \omega(R, R)\right)(\Gamma+|\Theta|)(t)+o(\Gamma+|\Theta|) .
$$

Let us point out that all the $o(\Gamma+|\Theta|)$-terms can be made uniformly small in the interval $[0, T]$ by taking $\varepsilon_{0}$ small by their definitions and using that $\operatorname{supp}\left(\hat{\mu}_{t}\right) \subset\left[R-4 \varepsilon_{0}, R+4 \varepsilon_{0}\right]$ in $[0, T]$. More precisely, let $\gamma \in\left(0,-\max \left(\partial_{1} \omega(R, R), \frac{d}{d R} \omega(R, R)\right)\right)$. We can choose $\varepsilon_{0}>0$ small enough for the rest terms of (27) to satisfy:

$$
\frac{o(\Gamma(t)+|\Theta(t)|)}{\Gamma(t)+|\Theta(t)|} \leq\left|\max \left(\partial_{1} \omega(R, R), \frac{d}{d R} \omega(R, R)\right)\right|-\gamma
$$

for any $\Gamma(t), \Theta(t)$ since $\Gamma(t)+|\Theta(t)| \leq 4 \varepsilon_{0}$ for all $t \in[0, T]$ due to (25). Then (28) is satisfied for all $t \in[0, T]$, and thus,

$$
\frac{d}{d t}(\Gamma+|\Theta|)(t) \leq-\gamma(\Gamma+|\Theta|)(t)
$$


so that for $t \in[0, T]$,

$$
(\Gamma+|\Theta|)(t) \leq(\Gamma+|\Theta|)(0) e^{-\gamma t}
$$

In particular, for any time $t \in[0, T],(\Gamma+|\Theta|)(t) \leq(\Gamma+|\Theta|)(0) \leq 3 \varepsilon_{0}$ and thus, using the continuity of $(\Gamma+|\Theta|)(t)$ since $T<+\infty$ we can continue up to $\tilde{T}>T$ satisfying $(\Gamma+|\Theta|)(t) \leq 4 \varepsilon_{0}$ contradicting the definition of $T$. Thus, $T=\infty$ for small enough $\varepsilon_{0}$ and $(29)$ then holds for all $t \geq 0$. Thanks to $(25)$, this implies the exponential convergence of $d_{2}\left(\hat{\mu}_{t}, \delta_{R}\right)$ to 0 :

$d_{2}\left(\hat{\mu}_{t}, \delta_{R}\right)^{2}=\int_{0}^{\infty}(r-R)^{2} d \hat{\mu}_{t}(r) \leq \max \left(\left|r_{1}(t)-R\right|^{2},\left|r_{2}(t)-R\right|^{2}\right) \leq(\Gamma+|\Theta|)^{2}(t) \leq 3 \varepsilon_{0} e^{-\gamma t}$

for all $t \geq 0$.

Remark 5. Lemma 1 gives sufficient conditions to get the assume regularity $\omega \in C^{1}\left(\mathbb{R}_{+}^{2}\right)$. Previous Theorem holds for all radially symmetric potentials $W(x)=k(|x|)$ belonging to $C^{3}\left(\mathbb{R}^{N} \backslash\{0\}\right)$ such that $k^{\prime \prime}(r)$ and $r^{-1} k^{\prime}(r)$ are integrable on hypersurfaces. This applies also to the next result for non local perturbations.

Remark 6. The first part of condition (24) implies intuitively that the velocity field created by $\delta_{R}$ given by $\omega(r, R)$ is decreasing at $r=R$ and therefore, particles are pushed locally in space and in time towards radius $R$ for small perturbations.

From now on, we denote by $\varphi(t, \cdot)$ the pseudo-inverse of the distribution function of the radial measure $\hat{\mu}_{t}$, that is

$$
\varphi(t, \xi)=\inf \left\{r \in \mathbb{R}_{+} ; \int_{0}^{r} d \hat{\mu}_{t} \geq \xi\right\} .
$$

$\varphi$ then satisfies

$$
\partial_{t} \varphi(t, \xi)=\hat{v}(t, \varphi(t, \xi))=\int_{0}^{\infty} \omega(\varphi(t, \xi), \eta) d \hat{\mu}_{t}(\eta)
$$

Note that by the definition of $\varphi$,

$$
\int_{\left[r_{1}, r_{2}\right]} d \hat{\mu}_{t}(\eta)=\int_{\left\{\xi ; r_{1} \leq \varphi(t, \xi) \leq r_{2}\right\}} d \xi
$$

In the next theorem, we will work with solutions to system (5)-(6) satisfying (HMR) for which the pseudo-inverse of the distribution function is an absolutely continuous function on time satisfying (31) in the classical sense a.e. in $t$. Solutions obtained in Section 5 do satisfy these conditions.

Theorem 6 (Stability: Tail control). Assume $\omega \in C^{1}\left(\mathbb{R}^{2}\right)$ and that $\delta_{R}$ is a locally-stable stationary solution to (5)-(6), that is, $\omega(R, R)=0$ and the local stability condition (24) holds. Assume moreover that the velocity field associated to $\delta_{R}$ verifies

$$
\omega(r, R)>0 \text { on }(0, R), \quad \omega(r, R)<0 \text { on }(R, \infty), \quad \text { and } \quad \partial_{1} \omega(0, R)>0,
$$

and the following long-range controls on the interaction potential: for some $\alpha \geq 1$, there exists $\lambda>0$ such that

$$
\begin{gathered}
\omega(r, \eta) \leq \frac{1}{\lambda}-\lambda r^{\alpha} \text { for }(r, \eta) \in \mathbb{R}_{+} \times[R-\lambda, R+\lambda], \\
\sup _{[0, \lambda]}\left|\partial_{1} \omega(\cdot, \eta)\right| \leq \frac{1}{\lambda}\left(1+\eta^{\alpha}\right),
\end{gathered}
$$




$$
|\omega(r, \eta)| \leq \frac{1}{\lambda}\left(1+r^{\alpha}\right)\left(1+\eta^{\alpha}\right) \text { for }(r, \eta) \in \mathbb{R}_{+}^{2} .
$$

Then, for any solution to (5)-(6) satisfying (HMR) and (31) with initial data $\mu_{0} \in \mathcal{P}_{2}^{r}\left(\mathbb{R}^{N}\right)$ such that $\hat{\mu}_{0}(\{0\})=0$, and $d_{\alpha}\left(\hat{\mu}_{0}, \delta_{R}\right)$ is small enough,

$$
\lim _{t \rightarrow \infty} d_{\alpha}\left(\hat{\mu}_{t}, \delta_{R}\right)=0
$$

Remark 7. If we assume that the initial condition is compactly supported, then the longrange controls (33), (34), (35) on the interaction potential are not required anymore. Those are only necessary to control the behavior of the tail of the distribution and its interaction with the rest.

\section{Proof of the Theorem.}

Step 1.- "Claim: Given $\hat{\mu} \in \mathcal{P}_{2}^{r}\left(\mathbb{R}^{N}\right)$. If $d_{\alpha}\left(\hat{\mu}, \delta_{R}\right)$ is small, then the associated velocity fields to $\hat{\mu}$ and $\delta_{R}$ share some confining properties": For any $\vartheta>0$ small enough, thanks to our assumptions on $\omega$, we can show that there exists $\Lambda>0$ such that if $d_{\alpha}\left(\hat{\mu}, \delta_{R}\right) \leq \Lambda$, then

$$
\left\{\begin{array}{l}
\hat{v}(r)>C_{1} r>0 \text { on }(0, \vartheta], \\
\hat{v}(r)>v_{1}>0 \text { on }[\vartheta, R-\vartheta], \\
\hat{v}(r)<-v_{1} \text { on }[R+\vartheta, \infty),
\end{array}\right.
$$

where $\hat{v}(r)$ is the velocity field associated to $\hat{\mu}$ by $(6)$. To prove the first inequality, notice that $d_{\alpha}\left(\hat{\mu}, \delta_{R}\right) \leq \Lambda$ implies that

$$
\int_{[R-\sqrt{\Lambda}, R+\sqrt{\Lambda}]^{c}} d \hat{\mu}(\eta) \leq \Lambda^{-\alpha / 2} \int_{[R-\sqrt{\Lambda}, R+\sqrt{\Lambda}]^{c}}|\eta-R|^{\alpha} d \hat{\mu}(\eta) \leq \Lambda^{\frac{\alpha}{2}}
$$

is small. We can then estimate the velocity field $v$ for $0 \leq r \leq \vartheta \leq \lambda$ :

$$
\begin{aligned}
\hat{v}(r) & =\int_{0}^{\infty} \omega(r, \eta) d \hat{\mu}(\eta)=\int_{0}^{\infty}\left[\omega(0, \eta)+r \partial_{1} \omega(\theta, \eta)\right] d \hat{\mu}(\eta) \\
& =r \int_{[R-\sqrt{\Lambda}, R+\sqrt{\Lambda}]} \partial_{1} \omega(\theta, \eta) d \hat{\mu}(\eta)+r \int_{[R-\sqrt{\Lambda}, R+\sqrt{\Lambda}]^{c}} \partial_{1} \omega(\theta, \eta) d \hat{\mu}(\eta) .
\end{aligned}
$$

Note that $\omega(0, \eta)$ is equal to zero by definition. We then use (34) to get the following estimate:

$$
\begin{aligned}
\left|\int_{[R-\sqrt{\Lambda}, R+\sqrt{\Lambda}]^{c}} \partial_{1} \omega(\theta, \eta) d \hat{\mu}(\eta)\right| & \leq \int_{[R-\sqrt{\Lambda}, R+\sqrt{\Lambda}]^{c}} C\left(1+\eta^{\alpha}\right) d \hat{\mu}(\eta) \\
& \leq C\left(\Lambda^{\frac{\alpha}{2}}+d_{\alpha}\left(\hat{\mu}, \delta_{R}\right)^{\alpha}\right) \leq C \Lambda^{\alpha / 2} .
\end{aligned}
$$

Now, if $\Lambda$ is small enough and $r \in[0, \vartheta]$, then thanks to (37) and an argument as in (27) we conclude

$$
\hat{v}(r) \geq r \partial_{1} \omega(0, R)\left(1-\int_{[R-\sqrt{\Lambda}, R+\sqrt{\Lambda}]^{c}} d \hat{\mu}(\eta)-C r \Lambda^{1 / 2}\right)-C r \Lambda^{\alpha / 2} \geq \frac{\partial_{1} \omega(0, R)}{2} r .
$$


The second inequality (36) comes directly from assumption (35) and the continuity of $\omega$ : for $r \in[\vartheta, R-\vartheta]$ and $\Lambda$ small enough,

$$
\begin{aligned}
\hat{v}(r)= & \int_{[R-\sqrt{\Lambda}, R+\sqrt{\Lambda}]} \omega(r, \eta) d \hat{\mu}(\eta)+\int_{[R-\sqrt{\Lambda}, R+\sqrt{\Lambda}]^{c}} \omega(r, \eta) d \hat{\mu}(\eta) \\
\geq & \left(\omega(r, R)-\sqrt{\Lambda}\left\|\partial_{2} \omega\right\|_{L^{\infty}([\vartheta, R-\vartheta] \times[R-\sqrt{\Lambda}, R+\sqrt{\Lambda}])}\right) \int_{[R-\sqrt{\Lambda}, R+\sqrt{\Lambda}]} d \hat{\mu}(\eta) \\
& -C \int_{[R-\sqrt{\Lambda}, R+\sqrt{\Lambda}]^{c}}\left(1+\eta^{\alpha}\right) d \hat{\mu}(\eta) \\
\geq & \frac{1}{2} \omega(r, R)-C \Lambda^{\alpha / 2}
\end{aligned}
$$

where we have used $(37)$. Since $\omega(\cdot, R)>0$ on $(0, R)$, if $\Lambda>0$ is small enough, $v(r)>0$ on $[\vartheta, R-\vartheta]$.

For the last inequality in (36), we can write the velocity field as

$$
\hat{v}(r)=\int_{[R-\sqrt{\Lambda}, R+\sqrt{\Lambda}]} \omega(r, \eta) d \hat{\mu}(\eta)+\int_{[R-\sqrt{\Lambda}, R+\sqrt{\Lambda}]^{c}} \omega(r, \eta) d \hat{\mu}(\eta)
$$

and estimate the second term of (38) using (35) and (37) to obtain

$$
\left|\int_{[R-\sqrt{\Lambda}, R+\sqrt{\Lambda}]^{c}} \omega(r, \eta) d \hat{\mu}(\eta)\right| \leq C\left(1+r^{\alpha}\right) \int_{[R-\sqrt{\Lambda}, R+\sqrt{\Lambda}]^{c}}\left(1+\eta^{\alpha}\right) d \hat{\mu}(\eta) \leq C \Lambda^{\alpha / 2}\left(1+r^{\alpha}\right) .
$$

Let us distinguish two cases. In the set $r \geq\left(\frac{1}{\lambda}\left(\frac{1}{\lambda}-1\right)\right)^{1 / \alpha}$ which is equivalent to $\frac{1}{\lambda}-\lambda r^{\alpha} \leq-1$, we deduce that there exists $C_{1}>0$ such that $\frac{1}{\lambda}-\lambda r^{\alpha} \leq-C_{1}\left(1+r^{\alpha}\right)$. We can then control the first term of (38) using (33) and (37) to get

$$
\begin{aligned}
\int_{[R-\sqrt{\Lambda}, R+\sqrt{\Lambda}]} \omega(r, \eta) d \hat{\mu}(\eta) & \leq\left(\frac{1}{\lambda}-\lambda r^{\alpha}\right) \int_{[R-\sqrt{\Lambda}, R+\sqrt{\Lambda}]} d \hat{\mu} \\
& \leq-C_{1}\left(1+r^{\alpha}\right)\left(1-\int_{[R-\sqrt{\Lambda}, R+\sqrt{\Lambda}]^{c}} d \hat{\mu}\right) \leq-\frac{C_{1}}{2}\left(1+r^{\alpha}\right) .
\end{aligned}
$$

For $r \in I:=\left[R+\vartheta,\left(\frac{1}{\lambda}\left(\frac{1}{\lambda}-1\right)\right)^{1 / \alpha}\right]$, we use the assumption that $\omega(\cdot, R)<0$ on the compact interval $I$. By continuity of $\omega$, we thus have that for $\Lambda>0$ small enough and $r \in I$,

$$
\max \{\omega(r, \eta) ; r \in I, \eta \in[R-\sqrt{\Lambda}, R+\sqrt{\Lambda}]\}:=-C_{2}<0,
$$

and thus,

$$
\int_{[R-\sqrt{\Lambda}, R+\sqrt{\Lambda}]} \omega(r, \eta) d \hat{\mu}(\eta) \leq-C_{2} \int_{[R-\sqrt{\Lambda}, R+\sqrt{\Lambda}]} d \hat{\mu} \leq-\frac{C_{2}}{2} \leq-C_{3}\left(1+r^{\alpha}\right)<0,
$$

for $r \in I$ and $\Lambda$ small enough using (37). Then, (38) becomes

$$
\hat{v}(r) \leq\left(-\min \left(C_{1}, C_{3}\right)+C \sqrt{\Lambda}\right)\left(1+r^{\alpha}\right) \leq-C_{4}\left(1+r^{\alpha}\right),
$$

for any $r \geq R+\vartheta$, if $\Lambda>0$ is small enough. 
Step 2.- "Claim: If $\mu_{0}$ is close enough to $\delta_{R}$, then $\hat{\mu}_{t}$ satisfies (36) at all times." Let $\varphi(t, \xi)$ the associated pseudo-inverse function associated to $\hat{\mu}_{t}$ by (30). We assume that $\hat{\mu}_{0}$ satisfies

$$
d_{\alpha}\left(\delta_{R}, \hat{\mu}_{0}\right)<\varepsilon .
$$

For any $\varepsilon>0$, we can estimate $|\varphi(0, \sqrt{\varepsilon})-R|$ as follows:

$$
\begin{aligned}
\varepsilon & \geq d_{\alpha}\left(\delta_{R}, \hat{\mu}_{0}\right) \geq d_{1}\left(\delta_{R}, \hat{\mu}_{0}\right)=\int_{0}^{1}|\varphi(0, \xi)-R| d \xi \\
& \geq \max \left(\int_{0}^{\sqrt{\varepsilon}}|\varphi(0, \xi)-R| d \xi, \int_{\sqrt{\varepsilon}}^{1}|\varphi(0, \xi)-R| d \xi\right) .
\end{aligned}
$$

Since $\varphi$ is not decreasing, if $\varphi(0, \sqrt{\varepsilon}) \leq R$, then $|\varphi(0, \xi)-R| \geq|\varphi(0, \sqrt{\varepsilon})-R|$ for $\xi \in[0, \sqrt{\varepsilon}]$. If $\varphi(0, \sqrt{\varepsilon}) \geq R$, then $|\varphi(0, \xi)-R| \geq|\varphi(0, \sqrt{\varepsilon})-R|$ for $\xi \in[\sqrt{\varepsilon}, 1]$, so that

$$
\varepsilon \geq \min (\sqrt{\varepsilon}|\varphi(0, \sqrt{\varepsilon})-R|,(1-\sqrt{\varepsilon})|\varphi(0, \sqrt{\varepsilon})-R|),
$$

which provides the estimate $|\varphi(0, \sqrt{\varepsilon})-R| \leq \sqrt{\varepsilon}$. Similarly, $|\varphi(0,1-\sqrt{\varepsilon})-R| \leq \sqrt{\varepsilon}$, so that

$$
\varphi(0,[\sqrt{\varepsilon}, 1-\sqrt{\varepsilon}]) \subset[R-\sqrt{\varepsilon}, R+\sqrt{\varepsilon}] .
$$

Let us define $\Gamma_{\varepsilon}(t):=\varphi(t, 1-\sqrt{\varepsilon})-\varphi(t, \sqrt{\varepsilon})$ and

$$
\Theta_{\varepsilon}(t):=\frac{1}{1-2 \sqrt{\varepsilon}} \int_{\sqrt{\varepsilon}}^{1-\sqrt{\varepsilon}} \varphi(t, \xi) d \xi-R .
$$

Notice that for $\xi \in[\sqrt{\varepsilon}, 1-\sqrt{\varepsilon}]$,

$$
|\varphi(t, \xi)-R| \leq \Gamma_{\varepsilon}(t)+\Theta_{\varepsilon}(t) .
$$

For $0<\varepsilon \leq \varepsilon_{0} \leq \Lambda$, we define $T:=\min \left\{t \in[0, \tau] ; \Gamma_{\varepsilon}(t)+\left|\Theta_{\varepsilon}(t)\right| \geq \varepsilon_{0}\right.$ or $\left.d_{\alpha}\left(\delta_{R}, \hat{\mu}_{t}\right) \geq \varepsilon_{0}\right\}$. Thanks to (39), $T>0$ by continuity for $0<\varepsilon<\min \left(\Lambda, \varepsilon_{0}\right)$ small enough. We will show that there exists $\varepsilon>0$ such that $T=+\infty$. Assume by contradiction that $T<\infty$. By definition of $T$, we have $d_{\alpha}\left(\delta_{R}, \hat{\mu}_{t}\right) \leq \varepsilon_{0} \leq \Lambda$ for $t \in[0, T]$.

Thus, $\hat{\mu}_{t}$ satisfies $(36)$ for $t \in[0, T], v(t, \cdot)$ is positive on $[0, R-\vartheta]$ and negative on $[R+\vartheta, \infty)$, and then (31) implies that $\xi \in[0,1],|\varphi(t, \xi)-R| \leq \max (|\varphi(0, \xi)-R|, \vartheta)$. In particular, by (37) we get

$$
\int_{[\sqrt{\varepsilon}, 1-\sqrt{\varepsilon}]^{c}}|\varphi(t, \xi)-R|^{\alpha} d \xi \leq \int_{[\sqrt{\varepsilon}, 1-\sqrt{\varepsilon}]^{c}}\left[|\varphi(0, \xi)-R|^{\alpha}+\vartheta^{\alpha}\right] d \xi \leq C \sqrt{\varepsilon} .
$$

For $t \in[0, T]$, we deduce that

$$
\begin{aligned}
\frac{d}{d t} \Gamma_{\varepsilon}(t)= & \hat{v}(t, \varphi(t, 1-\sqrt{\varepsilon}))-\hat{v}(t, \varphi(t, \sqrt{\varepsilon})) \\
= & \int_{[\varphi(t, \sqrt{\varepsilon}), \varphi(t, 1-\sqrt{\varepsilon})]}[\omega(\varphi(t, 1-\sqrt{\varepsilon}), \eta)-\omega(\varphi(t, \sqrt{\varepsilon}), \eta)] d \hat{\mu}_{t}(\eta) \\
& +\int_{[\varphi(t, \sqrt{\varepsilon}), \varphi(t, 1-\sqrt{\varepsilon})]^{c}}[\omega(\varphi(t, 1-\sqrt{\varepsilon}), \eta)-\omega(\varphi(t, \sqrt{\varepsilon}), \eta)] d \hat{\mu}_{t}(\eta) .
\end{aligned}
$$


The first term can be estimated as it has been done for $\Gamma(t)$ in the proof of Theorem 5 . To estimate the second term, we use (35), (40), and (41) to conclude that

$$
\begin{aligned}
& \left|\int_{[\varphi(t, \sqrt{\varepsilon}), \varphi(t, 1-\sqrt{\varepsilon})]^{c}}[\omega(\varphi(t, 1-\sqrt{\varepsilon}), \eta)-\omega(\varphi(t, \sqrt{\varepsilon}), \eta)] d \hat{\mu}_{t}(\eta)\right| \\
& \quad \leq \frac{C}{\lambda}\left[1+\min \left(\varphi(t, \sqrt{\varepsilon})^{\alpha}, \varphi(t, 1-\sqrt{\varepsilon})^{\alpha}\right)\right] \int_{[\varphi(t, \sqrt{\varepsilon}), \varphi(t, 1-\sqrt{\varepsilon})]^{c}}\left(1+\eta^{\alpha}\right) d \hat{\mu}_{t}(\eta) \\
& \quad \leq \frac{C}{\lambda}\left[1+o\left(\Gamma_{\varepsilon}+\left|\Theta_{\varepsilon}\right|\right)\right]\left[C \sqrt{\varepsilon}+\int_{[\sqrt{\varepsilon}, 1-\sqrt{\varepsilon}]^{c}}|\varphi(t, \xi)-R|^{\alpha} d \xi\right] \\
& \quad \leq C \sqrt{\varepsilon}+o\left(\Gamma_{\varepsilon}+\left|\Theta_{\varepsilon}\right|\right) .
\end{aligned}
$$

The same can be done for $\Theta_{\varepsilon}$, and we obtain

$$
\frac{d}{d t}\left(\Gamma_{\varepsilon}+\left|\Theta_{\varepsilon}\right|\right)(t) \leq \max \left(\partial_{1} \omega(R, R), \frac{d}{d R} \omega(R, R)\right)\left(\Gamma_{\varepsilon}+\left|\Theta_{\varepsilon}\right|\right)(t)+o\left(\Gamma_{\varepsilon}+\left|\Theta_{\varepsilon}\right|\right)+C \sqrt{\varepsilon} .
$$

As it has been done in the proof of Theorem 5, $\varepsilon_{0}$ can be chosen small enough such that this implies for $t \in[0, T]$, that

$$
\frac{d}{d t}\left(\Gamma_{\varepsilon}+\left|\Theta_{\varepsilon}\right|\right)(t) \leq-\gamma\left(\Gamma_{\varepsilon}+\left|\Theta_{\varepsilon}\right|\right)(t)+C \sqrt{\varepsilon}
$$

where $\gamma:=\frac{1}{2}\left|\max \left(\partial_{1} \omega(R, R), \frac{d}{d R} \omega(R, R)\right)\right|$. Then, for $t \in[0, T]$,

$$
\left(\Gamma_{\varepsilon}+\left|\Theta_{\varepsilon}\right|\right)(t) \leq \max \left(\left(\Gamma_{\varepsilon}+\left|\Theta_{\varepsilon}\right|\right)(0), \frac{C}{\gamma} \sqrt{\varepsilon}\right) \leq C \sqrt{\varepsilon}
$$

and

$$
\begin{aligned}
d_{\alpha}\left(\delta_{R}, \hat{\mu}_{t}\right)^{\alpha} & \leq\left[\left(\Gamma_{\varepsilon}+\left|\Theta_{\varepsilon}\right|\right)(t)\right]^{\alpha}+\int_{[\sqrt{\varepsilon}, 1-\sqrt{\varepsilon}]^{c}}|\varphi(t, \xi)-R|^{\alpha} d \xi \\
& \leq \max \left(\left(\Gamma_{\varepsilon}+\left|\Theta_{\varepsilon}\right|\right)(0), \frac{C}{\gamma} \sqrt{\varepsilon}\right)^{\alpha}+C \sqrt{\varepsilon} \leq C \sqrt{\varepsilon},
\end{aligned}
$$

due to $(39)$ and $(41)$.

If $\varepsilon>0$ is small enough, this implies that for $t \in[0, T],\left(\Gamma_{\varepsilon}+\left|\Theta_{\varepsilon}\right|\right)(t) \leq \frac{\varepsilon_{0}}{2}$, and $d_{\alpha}\left(\delta_{R}, \hat{\mu}(t)\right) \leq \frac{\varepsilon_{0}}{2}$. By a contradiction argument similar to the one used in the proof of Theorem 5. this shows that if $\varepsilon>0$ is small enough, then $T=+\infty$, and (36) is satisfied at all times.

Step 3.- "Claim: Asymptotic convergence of $\hat{\mu}_{t}$ to $\delta_{R}$ :" Since $(36)$ is satisfied for all $t \geq 0$, $\hat{v}(t, r) \geq C_{1} r$ on $[0, \vartheta]$, and then $\varphi(t, \xi) \geq \varphi(0, \xi) e^{C_{1} t}$ if $\varphi(t, \xi) \leq \vartheta$, due to (31). We can thus estimate, using (32):

$$
\begin{aligned}
\int_{0}^{\vartheta}\left(1+r^{\alpha}\right) d \hat{\mu}_{t}(r) & \leq\left(1+R^{\alpha}\right) \int_{0}^{\vartheta} d \hat{\mu}_{t}(r)=\left(1+R^{\alpha}\right) \int_{\{\xi ; \varphi(t, \xi) \leq \vartheta\}} d \xi \\
& \leq\left(1+R^{\alpha}\right) \int_{\left\{\xi ; \varphi(0, \xi) \leq \vartheta e^{-C_{1} t}\right\}} d \xi=\left(1+R^{\alpha}\right) \int_{0}^{\vartheta e^{-C_{1} t}} d \hat{\mu}_{0}(r) .
\end{aligned}
$$

Since (36) is satisfied, we claim that $\hat{v}(t, r) \geq v_{1}$ on $[\vartheta, R-\vartheta]$, and then $\varphi(t, \xi) \in[0, R-\vartheta]$ for $t \geq \frac{R}{v_{1}}$ implies that $\varphi\left(t-\frac{R}{v_{1}}, \xi\right) \leq \vartheta$. 
To see this, we make use of $(36)$ to get $\hat{v}(t, \cdot) \geq 0$ on $[0, R-\vartheta]$. If $\varphi(t, \xi) \in[0, R-\vartheta], \varphi(\cdot, \xi)$ is thus increasing on $[0, t]$. If $\varphi\left(t-\frac{R}{v_{1}}, \xi\right) \geq \vartheta$, then $\varphi\left(\left[t-\frac{R}{v_{1}}, t\right], \xi\right) \subset[\vartheta, R-\vartheta]$

$$
R>\varphi(t, \xi)-\varphi\left(t-\frac{R}{v_{1}}, \xi\right)=\int_{t}^{t-\frac{R}{v_{1}}} \hat{v}(\sigma, \varphi(\sigma, \xi)) d \sigma \geq \frac{R}{v_{1}} v_{1},
$$

which is absurd, thus $\varphi\left(t-\frac{R}{v_{1}}, \xi\right) \leq \vartheta$ as desired. We can then estimate, for $t \geq \frac{R}{v_{1}}$, using (32),

$$
\begin{aligned}
\int_{0}^{R-\vartheta}\left(1+r^{\alpha}\right) d \hat{\mu}_{t}(r) & \leq\left(1+R^{\alpha}\right) \int_{0}^{R-\vartheta} d \hat{\mu}_{t}(r)=\left(1+R^{\alpha}\right)\left(\int_{0}^{\vartheta} d \hat{\mu}_{t}(\xi)+\int_{\vartheta}^{R-\vartheta} d \hat{\mu}_{t}(\xi)\right) \\
& =\left(1+R^{\alpha}\right)\left(\int_{\{\xi ; \varphi(t, \xi) \in[0, \vartheta]\}} d \xi+\int_{\vartheta}^{R-\vartheta} d \hat{\mu}_{t}(\xi)\right) \\
& \leq\left(1+R^{\alpha}\right) \int_{\left\{\xi ; \varphi(0, \xi) \leq \vartheta e^{\left.-C_{1} t\right\}}\right.} d \xi+\left(1+R^{\alpha}\right) \int_{\left\{\xi ; \varphi\left(t-\frac{R}{v_{1}}, \xi\right) \leq \vartheta\right\}} d \xi \\
& =\left(1+R^{\alpha}\right) \int_{0}^{\vartheta e^{-C_{1} t}} d \hat{\mu}_{0}(r)+\left(1+R^{\alpha}\right) \int_{0}^{\vartheta} d \hat{\mu}_{t-R / v_{1}}(r) .
\end{aligned}
$$

Now, using a similar argument as in 42 since $\varphi\left(t-\frac{R}{v_{1}}, \xi\right) \leq \vartheta$ in the last integral, we get

$$
\int_{0}^{R-\vartheta}\left(1+r^{\alpha}\right) d \hat{\mu}_{t}(r) \leq 2\left(1+R^{\alpha}\right) \int_{0}^{\vartheta e^{-C_{1}\left(t-R / v_{1}\right)}} d \hat{\mu}_{0}(r)
$$

Thanks to (36), if $\varphi(t, \xi) \geq R+\vartheta$, then

$$
\varphi(t, \xi)=\varphi(0, \xi)+\int_{0}^{t} \hat{v}(s, \varphi(s, \xi)) d s \leq \varphi(0, \xi)-v_{1} t
$$

In particular, $\varphi(t, \xi)^{\alpha} \leq \varphi(0, \xi)^{\alpha}$ and, thanks to (31), we get

$$
\begin{aligned}
\int_{R+\vartheta}^{\infty}\left(1+r^{\alpha}\right) d \hat{\mu}_{t}(r) & =\int_{\{\xi ; \varphi(t, \xi) \geq R+\vartheta\}}\left(1+\varphi(t, \xi)^{\alpha}\right) d \xi \leq \int_{\left\{\xi ; \varphi(0, \xi) \geq R+\vartheta+v_{1} t\right\}}\left(1+\varphi(0, \xi)^{\alpha}\right) d \xi \\
& =\int_{R+\vartheta+v_{1} t}^{\infty}\left(1+r^{\alpha}\right) d \hat{\mu}_{0}(r) .
\end{aligned}
$$

Let $\varepsilon>0$. Thanks to (43), 44, there exists $\tau \geq 0$ such that for any $t \geq \tau$,

$$
\int_{[R-\vartheta, R+\vartheta]^{c}}\left(1+r^{\alpha}\right) d \hat{\mu}_{t}(r) \leq \sqrt{\varepsilon}
$$

Then, in particular, for any $t \geq \tau, \int_{0}^{R-\vartheta} d \hat{\mu}_{t}(r) \leq \sqrt{\varepsilon}$ and $\int_{R+\vartheta}^{\infty} d \hat{\mu}_{t}(r) \leq \sqrt{\varepsilon}$, that is $\varphi\left(t,[\sqrt{\varepsilon}, 1-\sqrt{\varepsilon}) \subset[R-\vartheta, R+\vartheta]\right.$ and $\left(\Gamma_{\varepsilon}+\left|\Theta_{\varepsilon}\right|\right)(t) \leq 3 \vartheta$ due to 40]. Now, for $t \geq \tau$, with an argument similar to the one used in Step 2, we get

$$
\frac{d}{d t}\left(\Gamma_{\varepsilon}+\left|\Theta_{\varepsilon}\right|\right)(t) \leq \max \left(\partial_{1} \omega(R, R), \frac{d}{d R} \omega(R, R)\right)\left(\Gamma_{\varepsilon}+\left|\Theta_{\varepsilon}\right|\right)(t)+o\left(\Gamma_{\varepsilon}+\left|\Theta_{\varepsilon}\right|\right)+C \sqrt{\varepsilon} .
$$


Since for any $t \geq \tau,\left.\varphi(t, \cdot)\right|_{[\sqrt{\varepsilon}, 1-\sqrt{\varepsilon}]}$ takes its values in the compact set $[R-\vartheta, R+\vartheta]$ independent of $\varepsilon$, we can apply an argument similar to the one used in Step 2. Choose $\vartheta>0$ such that (46) implies

$$
\frac{d}{d t}\left(\Gamma_{\varepsilon}+\left|\Theta_{\varepsilon}\right|\right)(t) \leq-\gamma\left(\Gamma_{\varepsilon}+\left|\Theta_{\varepsilon}\right|\right)(t)+C \sqrt{\varepsilon},
$$

and then, there exists some $T \geq \tau$ such that for $t \geq T$,

$$
\left(\Gamma_{\varepsilon}+\left|\Theta_{\varepsilon}\right|\right)(t) \leq 2 \frac{C \sqrt{\varepsilon}}{\gamma} .
$$

To conclude, we notice that thanks to 45$)$ and $(32)$,

$$
\begin{aligned}
\int_{[\sqrt{\varepsilon}, 1-\sqrt{\varepsilon}]^{c}}|\varphi(t, \xi)-R|^{\alpha} d \xi & \leq \int_{[\sqrt{\varepsilon}, 1-\sqrt{\varepsilon}]^{c}} \max \left(\vartheta^{\alpha},|\varphi(t, \xi)-R|^{\alpha}\right) d \xi \\
& \leq 2 \vartheta^{\alpha} \sqrt{\varepsilon}+\int_{\{\xi ;|\varphi(t, \xi)-R| \geq \vartheta\}}|\varphi(t, \xi)-R|^{\alpha} d \xi \\
& \leq 2 \vartheta^{\alpha} \sqrt{\varepsilon}+\int_{[R-\vartheta, R+\vartheta]^{c}}|r-R|^{\alpha} d \hat{\mu}_{t}(r) \\
& \leq C \sqrt{\varepsilon},
\end{aligned}
$$

which, together with (47), implies that for $t \geq T$,

$$
\begin{aligned}
d_{\alpha}\left(\hat{\mu}_{t}, \delta_{R}\right)^{\alpha} & =\int_{[\sqrt{\varepsilon}, 1-\sqrt{\varepsilon}]}|\varphi(t, \xi)-R|^{\alpha} d \xi+\int_{[\sqrt{\varepsilon}, 1-\sqrt{\varepsilon}]^{c}}|\varphi(t, \xi)-R|^{\alpha} d \xi \\
& \leq C \sqrt{\varepsilon} .
\end{aligned}
$$

Since this is true for any $\varepsilon>0$, it shows that $d_{\alpha}\left(\hat{\mu}_{t}, \delta_{R}\right) \rightarrow 0$ as $t \rightarrow \infty$.

\section{EXISTENCE THEORY}

Existence and uniqueness of weak solutions for the aggregation equation in $\mathcal{P}_{2}\left(\mathbb{R}^{N}\right) \cap$ $L^{p}\left(\mathbb{R}^{N}\right)$ have been given in [40, 7, 11]. Weak measure solutions to the the Cauchy problem for the aggregation equation (1) where given in [18] under the condition that the potential is smooth except possibly at the origin, the growth at infinity is no worse than quadratic, and the singularity at the origin of the derivative of the potential is not worse that Lipschitz. This section is aimed to give an existence theory of classical solutions for the aggregation equation.

We will denote by $\mathcal{W}^{m, p}\left(\mathbb{R}^{N}\right), 1 \leq p \leq \infty$ and $m \in \mathbb{N}$, the Sobolev spaces.

Theorem 7 (Existence of classical solutions). Let $W$ satisfy

$$
\nabla W \in L^{1}\left(\mathbb{R}^{N}\right), \quad D^{2} W \in L^{1}\left(\mathbb{R}^{N}\right), \quad(\Delta W)_{+} \in L^{\infty}\left(\mathbb{R}^{N}\right)
$$

Then, for any initial data $\rho_{0}(x) \in \mathcal{W}^{2, \infty}\left(\mathbb{R}^{N}\right)$, there exist classical solutions $\rho \in C^{1}([0, T] \times$ $\left.\mathbb{R}^{N}\right) \cap \mathcal{W}_{\text {loc }}^{1, \infty}\left(\mathbb{R}_{+}, \mathcal{W}^{1, \infty}\left(\mathbb{R}^{N}\right)\right)$ to (1). Moreover, if $\rho_{0}(x) \in \mathcal{W}^{\kappa, \infty}\left(\mathbb{R}^{N}\right)$ for $\kappa \in \mathbb{N}, \kappa \geq 2$, then $\rho \in C^{1}\left(\left(\mathbb{R}^{N}\right) \times[0, T]\right) \cap \mathcal{W}_{\text {loc }}^{\kappa-1, \infty}\left(\mathbb{R}_{+}, \mathcal{W}^{\kappa-1}\left(\mathbb{R}^{N}\right)\right)$. Furthermore, assuming in addition that $\rho_{0} \in L^{1}\left(\mathbb{R}^{N}\right)$ with bounded second moment, the solution is unique.

Proof of the theorem. Step 1: A priori estimates. In this step we assume that the solution is smooth as needed. This assumption will be removed in the next step. 
We consider first $x \in \mathbb{R}^{N}$ such that $\rho(x, t)=\|\rho(t)\|_{\infty}$. Then, $\nabla \rho(x, t)=0$, and

$$
\begin{aligned}
\partial_{t} \rho(x, t) & =\nabla \rho(x, t)(\nabla W * \rho)(x, t)+\rho(x, t)(\Delta W * \rho)(x, t) \\
& \leq \rho(x, t)\left((\Delta W * \rho)_{+}(x, t)\right) \\
& \leq\left\|(\Delta W)_{+}\right\|_{L^{\infty}} \rho(x, t),
\end{aligned}
$$

so that

$$
\|\rho(t)\|_{\infty} \leq\left\|\rho_{0}\right\|_{\infty} e^{\left\|(\Delta W)_{+}\right\|_{L} t}
$$

Let now $K \in \mathbb{N}, K \leq \kappa, i=\left(i_{1}, \ldots, i_{N}\right) \in \mathbb{N}^{N}$ and $x \in \mathbb{R}^{N}$ be such that $\sum_{j=1}^{N} i_{j}=K$, $\left|\partial_{i_{1}} \ldots \partial_{i_{N}} \rho(x, t)\right|=\left\|\partial_{i_{1}} \ldots \partial_{i_{N}} \rho(t)\right\|_{\infty}$, and we define

$$
\left\|D^{K} \rho\right\|_{p}=\sup \left\{\left\|\partial_{\sigma} \rho\right\|_{L^{p}},|\sigma| \leq K\right\}, \quad 1 \leq p \leq \infty, K \leq \kappa,
$$

where $\sigma=\left(\sigma_{1}, \ldots, \sigma_{N}\right) \in \mathbb{N}^{N}$. W.l.o.g. we suppose that $\partial_{i_{1}} \ldots \partial_{i_{N}} \rho(x, t) \geq 0$ (to change the sign of this term, one just needs to replace the element $e_{1}$ of the basis of $\mathbb{R}^{N}$ by $-e_{1}$ ), and then,

$$
\begin{aligned}
\partial_{t} \partial_{i_{1}} \ldots \partial_{i_{N}} \rho(x, t)= & \partial_{i_{1}} \ldots \partial_{i_{N}} \nabla_{x} \cdot(\rho(\nabla W * \rho))(x, t) \\
= & \sum_{k=0}^{K} \sum_{\substack{|\sigma|=k \\
\sigma \leq i \\
\sigma \leq i}}\left(\partial_{\sigma} \nabla \rho(x, t)\right) \cdot\left(\partial_{\sigma^{c}}(\nabla W * \rho)(x, t)\right) \\
& +\sum_{k=0}^{K} \sum_{\substack{\sigma \mid=k \\
\sigma \leq i}}\left(\partial_{\sigma} \rho(x, t)\right)\left(\partial_{\sigma^{c}}(\Delta W * \rho)(x, t)\right) .
\end{aligned}
$$

Here, $\sigma \leq i$ denotes $\sigma_{j} \leq i_{j}$ for $j=1, \ldots, N$ and $\sigma^{c}=\sigma-i$. Using that the term $k=K$ in the first sum is zero one obtains

$$
\begin{aligned}
\partial_{t} \partial_{i_{1}} \ldots \partial_{i_{N}} \rho(x, t)= & \nabla \rho(x, t) \cdot\left(\partial_{i_{1}} \nabla W\right) *\left(\partial_{i_{2}} \ldots \partial_{i_{N}} \rho(x, t)\right) \\
& +\rho(x, t)\left(\Delta W *\left(\partial_{i_{1}} \ldots \partial_{i_{N}} \rho(x, t)\right)(x, t)\right. \\
& +\left(\partial_{i_{1}} \ldots \partial_{i_{N}} \rho(x, t)\right)(\Delta W * \rho)(x, t) \\
& +\sum_{k=1}^{K-1} \sum_{\substack{|\sigma|=k \\
\sigma \leq i}}\left(\partial_{\sigma} \nabla \rho(x, t)\right)\left(\nabla W * \partial_{\sigma^{c}} \rho\right)(x, t) \\
& +\sum_{k=1}^{K-1} \sum_{\substack{|\sigma|=k \\
\sigma \leq i}}\left(\partial_{\sigma} \rho(x, t)\right)\left(\nabla W * \nabla \partial_{\sigma^{c}} \rho\right)(x, t),
\end{aligned}
$$


and then, we get the estimate:

$$
\begin{aligned}
\partial_{t} \partial_{i_{1}} \ldots \partial_{i_{N}} \rho(x, t) \leq & \|\nabla \rho\|_{\infty}\left\|\partial_{i_{1}} \nabla W\right\|_{L^{1}}\left\|\partial_{i_{2}} \ldots \partial_{i_{N}} \rho\right\|_{\infty} \\
& +\left\|\partial_{i_{1}} \ldots \partial_{i_{N}} \rho\right\|_{\infty}\|\Delta W\|_{L^{1}}\|\rho\|_{\infty} \\
& +\sum_{k=1}^{K-1} \sum_{\substack{|\sigma|=k \\
\sigma \leq i}}\left\|\partial_{\sigma} \nabla \rho\right\|_{\infty}\|\nabla W\|_{L^{1}}\left\|\partial_{\sigma^{c}} \rho\right\|_{\infty} \\
& +\sum_{k=1}^{K-1} \sum_{|\sigma|=k}\left\|\partial_{\sigma} \rho\right\|_{\infty}\|\nabla W\|_{L^{1}}\left\|\nabla \partial_{\sigma^{c}} \rho\right\|_{\infty} \\
\leq & \|\rho\|_{\infty}\|\Delta W\|_{L^{1}}\left\|\partial_{i_{1}} \ldots \partial_{i_{N}} \rho\right\|_{\infty}+\left\|\partial_{i_{1}} \nabla W\right\|_{L^{1}}\left\|\partial_{i_{2}} \ldots \partial_{i_{N}} \rho\right\|_{\infty}\|\nabla \rho\|_{\infty} \\
& +C_{K}\|\nabla W\|_{L^{1}}\left\|D^{K-1} \rho\right\|_{\infty}^{2}\left\|D^{K} \rho\right\|_{\infty} .
\end{aligned}
$$

An induction scheme on $K$ initialized by 49 then provides the following exponential control for $K \in\{0, \ldots, \kappa\}$ :

$$
\left\|D^{K} \rho\right\|_{\infty} \leq C_{1, K} e^{C_{2, K} t}
$$

where $C_{1, K}, C_{2, K}$ only depend on $K,\left\|D^{2} W\right\|_{L^{1}},\left\|(\Delta W)_{+}\right\|_{L^{\infty}}$, and $\left\|\rho_{0}\right\|_{\mathcal{W}^{\kappa, \infty}}$. Coming back to (50) the following estimate on the time derivative follows:

$$
\left\|\frac{d}{d t} D^{K} \rho\right\|_{\infty} \leq \tilde{C}_{1, K} e^{\tilde{C}_{2, K} t}
$$

Finally, taking the derivative in $t$ on (1) we get

$$
\begin{aligned}
\partial_{t}^{2} \rho= & \left(\nabla W * \partial_{t} \rho\right) \cdot(\nabla W * \rho)+\nabla_{x} \rho \cdot\left(\nabla W * \partial_{t} \rho\right) \\
& +\rho\left(\Delta W * \partial_{t} \rho\right)+\partial_{t} \rho(\Delta W * \rho),
\end{aligned}
$$

from which it is easy to derive

$$
\left\|\frac{d^{2}}{d t^{2}} \rho\right\|_{\infty} \leq C e^{C t}
$$

Step 2: Construction of a solution trough an approximation problem. Let $W^{\varepsilon}$ be a smooth approximation of $W$, that is $W^{\varepsilon} \in \mathcal{W}^{2, \infty}\left(\mathbb{R}^{N}\right)$ such that $\left\|D^{2} W^{\varepsilon}\right\|_{L^{1}},\left\|\left(\Delta W^{\varepsilon}\right)_{+}\right\|_{L^{\infty}}$ are uniformly bounded, and:

$$
\nabla W^{\varepsilon} \stackrel{\varepsilon \rightarrow 0}{\longrightarrow} \nabla W \quad \text { in } \quad L^{1}\left(\mathbb{R}^{N}\right) .
$$

Thanks to [40, 11, there exists a classical solution $\rho^{\varepsilon} \in \mathcal{W}_{\text {loc }}^{1, \infty}\left(\mathbb{R}_{+}, \mathcal{W}^{1, \infty}\left(\mathbb{R}^{N}\right)\right)$ with initial data $\rho_{0}$ for each regular interaction potential $W^{\varepsilon}$.

The estimate (51) provides a uniform bound on $\left\|\rho^{\varepsilon}\right\|_{\infty},\left\|\nabla \rho^{\varepsilon}\right\|_{\infty}$ and $\left\|D^{2} \rho^{\varepsilon}\right\|_{\infty}$. Since $\kappa \geq 2$ then (52) implies that $\frac{d}{d t} \rho^{\varepsilon}$ and $\left(\frac{d}{d t} \nabla \rho^{\varepsilon}\right)$ are uniformly bounded for $\varepsilon>0$. Applying the Ascoli-Arzelá theorem, due to (51), (52) and (53) there exist limits for $\rho^{\varepsilon}, \partial_{t} \rho^{\varepsilon}$ and $\nabla_{x} \rho^{\varepsilon}$ (where we have written $\varepsilon$ instead of $\left.\varepsilon_{k}\right)$ on $C([0, T] \times B)$ for any compact subset $B \subset \mathbb{R}^{N}$ and moreover the limits denoted by $\rho, \partial_{t} \rho$ and $\nabla_{x} \rho$ belong to $C\left([0, T] ; \mathcal{W}^{1, \infty}\left(\mathbb{R}^{N}\right)\right)$. For the velocity field $v^{\varepsilon}$ we have that

$$
\begin{aligned}
\left|v^{\varepsilon}(x, t)-v(x, t)\right| & \leq \int_{\mathbb{R}^{N}}\left|\left(\nabla W^{\varepsilon}-\nabla W\right)(x-y)\right| \rho^{\varepsilon}(y) d y+\int_{\mathbb{R}^{N}}|\nabla W(x-y)|\left|\rho^{\varepsilon}(y)-\rho(y)\right| d y \\
& =(I)_{\varepsilon}+(I I)_{\varepsilon} .
\end{aligned}
$$


For $(I)_{\varepsilon}$ one observes that

$$
\left|(I)_{\varepsilon}\right| \leq\left\|\nabla W^{\varepsilon}-\nabla W\right\|_{L^{1}}\left\|\rho^{\varepsilon}\right\|_{\infty} \rightarrow 0 \quad \text { as } \quad \varepsilon \rightarrow 0,
$$

and for $(I I)_{\varepsilon}$ one has that that

$$
|\nabla W(x-y)|\left|\rho^{\varepsilon}(y)-\rho(y)\right| \leq C|\nabla W(x-y)| \in L^{1}\left(\mathbb{R}^{N}\right) .
$$

Then, by the dominated convergence theorem, $(I I)_{\varepsilon} \rightarrow 0$ as $\varepsilon \rightarrow 0$ and, as a consequence, $v^{\varepsilon}(x, t)$ converges pointwise to $v(x, t)$ in $\mathbb{R}^{N} \times[0, T]$ for all $T>0$. The same reasoning is used to prove that $\nabla_{x} \cdot v^{\varepsilon} \rightarrow \nabla_{x} \cdot v$ as $\varepsilon \rightarrow 0$. Thus, the regularized equation

$$
\left.\frac{d}{d t} \rho^{\varepsilon}=\nabla \rho^{\varepsilon} \cdot\left(\nabla W^{\varepsilon} * \rho^{\varepsilon}\right)+\left(\Delta W^{\varepsilon} * \rho^{\varepsilon}\right) \rho^{\varepsilon}\right)
$$

passes to the limit and $\rho \in C^{1}\left([0, T] \times \mathbb{R}^{N}\right) \cap \mathcal{W}^{1, \infty}\left([0, T], \mathcal{W}^{1, \infty}\left(\mathbb{R}^{N}\right)\right)$.

The propagation of the regularity follows from estimates (51) and (52). The proof of uniqueness follows from [23, 11].

Remark 8. Under the assumptions on $W$ in the previous theorem we have that $\rho$ is Lipschitz continuous both in space and time, and then the characteristics are well defined:

$$
\frac{d}{d t} X_{t}=-(\nabla W * \rho)\left(X_{t}, t\right)
$$

and the solution $\rho$ is given by

$$
\rho(x, t)=\rho_{0}\left(X_{t}^{-1}\right) \operatorname{det}\left(D X_{t}^{-1}\right) .
$$

Remark 9. If $W$ and $\rho_{0}$ are radially symmetric, then one can easily check that the problem is invariant through rotations around the origin. The uniqueness result then shows that the solution $\rho$ is radially symmetric at all times. We also point out that if the solution is compactly supported then it remains of compact support for all times.

\section{The EXAmple of POWER LAW Repulsive-ATtRaCtive Potentials}

The aim of this section is to show an example of how to apply the general instability and stability theory in the case of power law repulsive-attractive potentials:

$$
W(x)=\frac{|x|^{a}}{a}-\frac{|x|^{b}}{b} \quad 2-N<b<a .
$$

The condition $b<a$ ensures that the potential is repulsive in the short range and attractive in the long range. One can easily check that for these type of potentials $\Delta W \in L_{l o c}^{\infty}\left(\mathbb{R}^{N}\right)$. The condition $2-N<b$ ensures that the potential is in $\mathcal{W}_{\text {loc }}^{1, q}\left(\mathbb{R}^{N}\right)$ for some $1<q<\infty$. Using algebraic computations, involving the Beta function, we give the conditions that the powers $a$ and $b$ should satisfy in order to apply the stability and instability theory, and we construct the bifurcation diagram for these powers. The main results of this section are the following:

Theorem 8 (Global existence of solutions for repulsive-attractive potentials). Given $W$ by (54). Assume $\rho_{0} \in \mathcal{W}^{2, \infty}\left(\mathbb{R}^{N}\right)$ is compactly supported and radially symmetric. Then there exists a global in time classical solution for (5)-(6). Furthermore, the solution is compactly supported and confined in a large ball for all times. 
Theorem 9 (Sharp radial stability-instability for spherical shells). Assume that $W$ is a power law potential as in (54). Then, there exists a unique $R_{a b}>0$ given by

$$
R_{a b}=\frac{1}{2}\left(\frac{\beta\left(\frac{b+N-1}{2}, \frac{N-1}{2}\right)}{\beta\left(\frac{a+N-1}{2}, \frac{N-1}{2}\right)}\right)^{\frac{1}{a-b}}
$$

such that $\delta_{R_{a b}}$ is stationary solution to (1). Moreover, the following properties hold:

(i) If $2-N<b \leq 3-N$ then $\omega \in C\left(\mathbb{R}_{+}^{2}\right) \cap C^{1}\left(\mathbb{R}_{+}^{2} \backslash \mathcal{D}\right)$ and for all $(R, R) \in \mathcal{D}$ we have

$$
\lim _{\substack{(r, \eta) \rightarrow(R, R) \\(r, \eta) \notin \mathcal{D}}} \frac{\partial \omega}{\partial r}(r, \eta)=+\infty .
$$

(ii) If $b \in\left(3-N, \frac{3 a-N a-10+7 N-N^{2}}{a+N-3}\right)$ then $\omega$ is $C^{1}\left(\mathbb{R}_{+}^{2}\right)$ and

$$
\partial_{1} \omega\left(R_{a b}, R_{a b}\right)>0 \text {. }
$$

(iii) If $b \in\left(\frac{3 a-N a-10+7 N-N^{2}}{a+N-3}, a\right)$ then $\omega$ is $C^{1}\left(\mathbb{R}_{+}^{2}\right)$ and

$$
\partial_{1} \omega\left(R_{a b}, R_{a b}\right)<0 \quad \text { and } \quad\left(\partial_{1} \omega+\partial_{2} \omega\right)\left(R_{a b}, R_{a b}\right)<0 .
$$

As a consequence, if $b \in\left(2-N, \frac{3 a-N a-10+7 N-N^{2}}{a+N-3}\right)$ then $\delta_{R_{a b}}$ is unstable in the sense of Theorem 3 and if $b \in\left(\frac{3 a-N a-10+7 N-N^{2}}{a+N-3}, a\right)$ then $\delta_{R_{a b}}$ is stable in the sense of Theorem 6 .

Remark 10. Note that indeed for $3-N<$ a we have

$$
3-N<\frac{3 a-N a-10+7 N-N^{2}}{a+N-3}<a .
$$

Remark 11. In [39] the authors study the dynamic of a curve evolving in $\mathbb{R}^{2}$ according to the aggregation equation. They perform a linear stability analysis of the spherical shell steady state. They consider not only radially symmetric perturbations but also perturbation which break the symmetry of the spherical shell. The mode $m=\infty$ corresponds to a perturbation which preserve the symmetry of the spherical shell. Using a computation involving the Gamma function, they show that the mode $m=\infty$ is stable if and only if $(a-1)(b-1)>1$. In order to prove (ii) we will perform similar type of computations involving special functions. Note that, when $N=2$

$$
\frac{3 a-N a-10+7 N-N^{2}}{a+N-3}=\frac{a}{a-1}
$$

so (ii) is equivalent to $(b-1)(a-1)>1$ and we recover the condition derived in [39].

As a summary of all the stability and instability results for a $\delta_{R_{a b}}$ stationary states for power law potentials we show the bifurcation diagram in Figure 1. For powers inside the region between $b=2-N$ and the curve one has instability of the $\delta_{R_{a b}}$. In the region above the curve one has stability of the $\delta_{R_{a b}}$. 


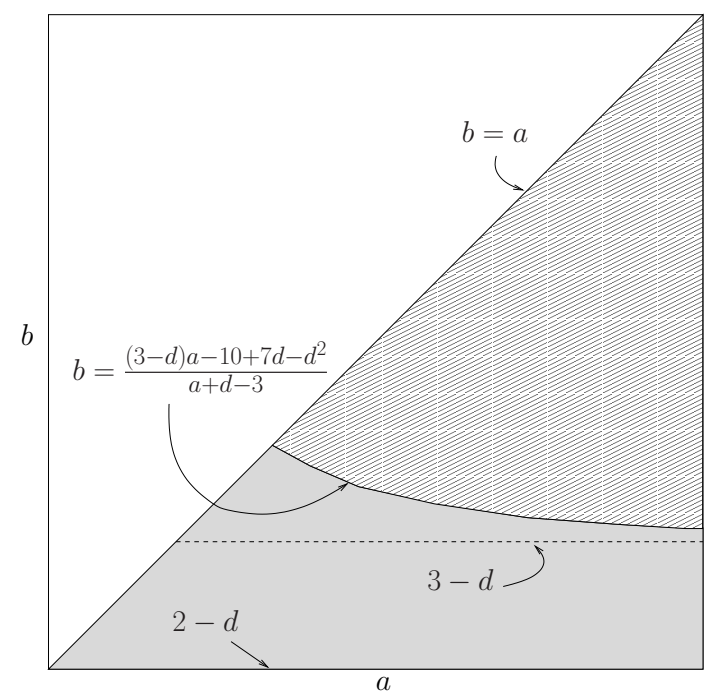

FiguRE 1. Bifurcation diagram. Stability and instability regions for $\delta_{R_{a b}}$.

6.1. Proof of Theorem 8. In order to prove the global existence theorem we need to introduce some notations. For potentials defined by (54), the kernel $\omega(r, \eta)$ defined in (2) becomes

$$
\begin{gathered}
\omega(r, \eta)=r^{b-1} \psi_{b}(\eta / r)-r^{a-1} \psi_{a}(\eta / r) \\
\psi_{a}(s)=\frac{1}{\sigma_{N}} \int_{\partial B(0, s)} \frac{\left(e_{1}-s y\right) \cdot e_{1}}{\left|e_{1}-s y\right|^{2-a}} d \sigma(y)
\end{gathered}
$$

The properties of the function $\psi_{a}(s)$ that we need are summarized in the following lemma and can be found in 30 .

Lemma 7 (Properties of the function $\left.\psi_{a}(s)\right)$. The function $\psi_{a}$ is continuous with $\psi_{a}(0)=1$ and $\lim _{s \rightarrow \infty} s^{2-a} \psi_{a}(s)=\frac{N+a-2}{N}$.

The main difficulty we have to cope with is the growth at infinity of the attractive part which restricts the range of direct application of Theorem 7.

Proof of Theorem 8. Due to translational invariance we can assume without loss of generality that the center of mass is located at zero. We write $W$ as $W(x)=W_{R}(x)+W_{A}(x)$ where $W_{A}(x)=\frac{|x|^{a}}{a}$ is the attractive part and $W_{R}(x)=-\frac{|x|^{b}}{b}$ is the repulsive part. In addition, since $W$ is radially symmetric, that is $W(x)=k(|x|)$ then we define $k(r):=k_{R}(r)+k_{A}(r)$. Finally, we write $\omega_{A}(r, \eta)=r^{a-1} \psi_{a}(\eta / r)$ and $\omega_{R}(r, \eta)=r^{b-1} \psi_{b}(\eta / r)$ with $\omega(r, \eta)=\omega_{R}(r, \eta)-\omega_{A}(r, \eta)$.

Step 1: A priori estimates on the support of $\hat{\rho}$. Suppose that $\hat{\rho}$ is a smooth radially symmetric solution for the equation (1) with compactly supported initial data $\rho_{0} \in \mathcal{W}^{2, \infty}\left(\mathbb{R}^{N}\right)$. Since the solutions belong to $\mathcal{W}_{\text {loc }}^{1, \infty}\left(\mathbb{R}_{+}, \mathcal{W}^{1, \infty}\left(\mathbb{R}^{N}\right)\right)$ and are compactly supported, the velocity field $\hat{v}$ is Lipschitz continuous in time and space and the characteristics are well defined. 
Thus $\hat{v}$ generates a $C^{1}$ flow map $r\left(t, r_{0}\right), t \in[0, T], r_{0} \in \mathbb{R}_{+}$:

$$
\begin{aligned}
\frac{d}{d t} r(t) & =\hat{v}\left(t, r\left(t, r_{0}\right)\right), \\
r\left(0, r_{0}\right) & =r_{0} .
\end{aligned}
$$

Let us define by $r_{2}(t)$ the characteristic curve starting at point $r_{2}(0)=\max \left\{\operatorname{supp}\left(\hat{\mu}_{0}\right)\right\}$. Then,

$$
\begin{aligned}
\frac{d}{d t} r_{2}(t) & =\hat{v}\left(t, r_{2}(t)\right)=\int_{0}^{\infty} \omega\left(r_{2}(t), \eta\right) d \hat{\mu}_{t}(\eta)=\int_{0}^{r_{2}(t)} \omega\left(r_{2}(t), \eta\right) d \hat{\mu}_{t}(\eta) \\
& =-\int_{0}^{r_{2}(t)} r_{2}(t)^{a-1} \psi_{a}\left(\eta / r_{2}(t)\right) d \hat{\mu}_{t}(\eta)+\int_{0}^{r_{2}(t)} r_{2}(t)^{b-1} \psi_{b}\left(\eta / r_{2}(t)\right) d \hat{\mu}_{t}(\eta),
\end{aligned}
$$

where we have used the expression of $\omega$ given in (55). Here $\hat{\mu}_{t}$ denotes the measure with density $\hat{\rho}_{t}$. Using the properties of $\psi_{a}$ in Lemma 7 in (57) we obtain the following inequality:

$$
\frac{d}{d t} r_{2}(t) \leq K_{b} r_{2}(t)^{b-1}-K_{a} r_{2}(t)^{a-1}
$$

where

$$
\left\{\begin{array}{lll}
K_{a}=1 & \text { and } \quad K_{b}=\psi_{b}(1), & \text { if } 2 \leq b<a, \\
K_{a}=1 & \text { and } \quad K_{b}=1, & \text { if } 2-N<b<2 \leq a, \\
K_{a}=\psi_{a}(1) & \text { and } \quad K_{b}=1, & \text { if } 2-N<b<a<2 .
\end{array}\right.
$$

Defining $\tilde{R}_{a b}:=\left(\frac{K_{a}}{K_{b}}\right)^{\frac{1}{b-a}}$ and rewriting (58) as

$$
\frac{d}{d t} r_{2}(t) \leq r_{2}(t)^{a-1}\left(K_{b} r_{2}(t)^{b-a}-K_{a}\right)
$$

one realizes that $r_{2}(t) \leq \bar{R}:=\max \left(r_{2}(0), \tilde{R}_{a b}\right)$ which proves that the $\operatorname{supp}\left(\hat{\mu}_{t}\right)$ is bounded and contained in $B(0, \bar{R})$ for all times.

Step 2: Global existence. Given $0<\varepsilon<1$, consider $\chi_{\varepsilon}(r)$ a $C^{\infty}(0, \infty)$ decreasing function with $0<\varepsilon<1$ such that $\chi_{\varepsilon}(r)=1$ if $0<r<1 / \varepsilon$ and $\chi_{\varepsilon}(r)=0$ if $r>1+1 / \varepsilon$. Define $f^{\varepsilon}(r):=\chi_{\varepsilon}(r) \cdot k_{A}^{\prime}(r) \in L^{1}(0, \infty), k_{A}^{\varepsilon}(r):=\int_{0}^{r} f^{\varepsilon}(s) d s$ and $k^{\varepsilon}(r)=k_{R}(r)+k_{A}^{\varepsilon}(r)$. Now, the potential $W^{\varepsilon}(x)=k^{\varepsilon}(|x|)$ satisfies the hypotheses of Theorem 7 so we have existence and uniqueness of classical solution $\hat{\rho}^{\varepsilon}$ to $(5)-(6)$ in $[0, T]$ with initial data $\hat{\rho}_{0}$. We denote by $\hat{\mu}_{t}^{\varepsilon}$ the measure with density $\hat{\rho}_{t}^{\varepsilon}$.

Consider $r_{2}=\max \left\{\operatorname{supp}\left(\hat{\mu}_{0}\right)\right\}$ and the characteristic curve $r_{2}^{\varepsilon}(t)$ starting at point $r_{2}=$ $r_{2}(0)$. Computing the derivative with respect to time, one has

$$
\begin{aligned}
\frac{d}{d t} r_{2}^{\varepsilon}(t) & =\int_{0}^{\infty} \omega^{\varepsilon}\left(r_{2}^{\varepsilon}(t), \eta\right) d \hat{\mu}_{t}^{\varepsilon}(\eta)=\int_{0}^{r_{2}^{\varepsilon}(t)} \omega^{\varepsilon}\left(r_{2}^{\varepsilon}(t), \eta\right) d \hat{\mu}_{t}^{\varepsilon}(\eta) \\
& =-\int_{0}^{r_{2}^{\varepsilon}(t)} \omega_{A}^{\varepsilon}\left(r_{2}^{\varepsilon}(t), \eta\right) d \hat{\mu}_{t}^{\varepsilon}(\eta)+\int_{0}^{r_{2}^{\varepsilon}(t)} r_{2}^{\varepsilon}(t)^{b-1} \psi_{b}\left(\eta / r_{2}^{\varepsilon}(t)\right) d \hat{\mu}_{t}^{\varepsilon}(\eta) \\
& \leq C_{b} r_{2}^{\varepsilon}(t)^{b-1}
\end{aligned}
$$

where we have split the kernel $\omega^{\varepsilon}$ into its attractive and repulsive parts, and we have used that $\omega_{A}^{\varepsilon} \geq 0$. The constant $C_{b}$ depends on $b$. The last inequality leads us to

$$
r_{2}^{\varepsilon}(t) \leq \sigma(t):=\left(C_{b}(2-b) t+r_{2}^{2-b}\right)^{\frac{1}{2-b}},
$$


which says that the solution exists at least up to time $T^{*}:=\frac{1}{2} \min \left\{T, T_{b}\right\}$. where

$$
T_{b}=\left\{\begin{array}{lll}
\frac{r_{2}(0)^{2-b}}{C_{b}(b-2)} & \text { if } \quad b>2, \\
+\infty & \text { if } \quad b \leq 2 .
\end{array}\right.
$$

In addition, (59) gives us a uniform estimate for the support of $\rho^{\varepsilon}$ up to time $T^{*}$. Notice that for all $t \leq T^{*}$ and $\varepsilon>0$ such that $2 \sigma\left(T^{*}\right) \varepsilon<1$, then $\nabla W^{\varepsilon} * \rho_{t}^{\varepsilon}=\nabla W * \rho_{t}^{\varepsilon}$ for all $x \in \operatorname{supp}\left(\rho_{t}^{\varepsilon}\right)$ and all $t \in\left[0, T^{*}\right]$. As a consequence $\omega$ given by (55) and $\omega^{\varepsilon}$ associated to $W^{\varepsilon}$ by (2) are equal in the set $\left\{(r, \eta) \mid(r, \eta) \in \operatorname{supp}\left(\rho_{t}^{\varepsilon}\right)^{2}\right\}$ for all $t \leq T^{*}$. Therefore, we can write:

$$
\begin{aligned}
\frac{d}{d t} r_{2}^{\varepsilon}(t) & =-\int_{0}^{r_{2}^{\varepsilon}(t)} \omega_{A}^{\varepsilon}\left(r_{2}^{\varepsilon}(t), \eta\right) d \hat{\mu}_{t}^{\varepsilon}(\eta)+\int_{0}^{r_{2}^{\varepsilon}(t)} r_{2}^{\varepsilon}(t)^{b-1} \psi_{b}\left(\eta / r_{2}^{\varepsilon}(t)\right) d \hat{\mu}_{t}^{\varepsilon}(\eta) \\
& =-\int_{0}^{r_{2}^{\varepsilon}(t)} \omega_{A}\left(r_{2}^{\varepsilon}(t), \eta\right) d \hat{\mu}_{t}^{\varepsilon}(\eta)+\int_{0}^{r_{2}^{\varepsilon}(t)} r_{2}^{\varepsilon}(t)^{b-1} \psi_{b}\left(\eta / r_{2}^{\varepsilon}(t)\right) d \hat{\mu}_{t}^{\varepsilon}(\eta) \\
& =-\int_{0}^{r_{2}^{\varepsilon}(t)} r_{2}^{\varepsilon}(t)^{a-1} \psi_{a}\left(\eta / r_{2}^{\varepsilon}(t)\right) d \hat{\mu}_{t}^{\varepsilon}(\eta)+\int_{0}^{r_{2}^{\varepsilon}(t)} r_{2}^{\varepsilon}(t)^{b-1} \psi_{b}\left(\eta / r_{2}^{\varepsilon}(t)\right) d \hat{\mu}_{t}^{\varepsilon}(\eta),
\end{aligned}
$$

and we can use the a priori estimates developed in Step 1. Then, for all $t \leq T^{*}$ we can conclude that $r_{2}^{\varepsilon}(t) \leq \bar{R}$. Now, let us take $\varepsilon$ such that $2 \varepsilon \bar{R}<1$. Therefore $\nabla W^{\varepsilon} * \rho_{t}^{\varepsilon}=\nabla W * \rho_{t}^{\varepsilon}$. For all $t \leq T^{*}$ in the support of $\rho_{t}^{\varepsilon}$. By uniqueness $\rho_{t}^{\varepsilon}=: \rho_{t}$ for all $2 \varepsilon \bar{R}<1$ and it is a classical solution to (1) with potential $W$. Summarizing, we have shown the existence of solution in the time interval $\left[0, T^{*}\right]$ with $r_{2}\left(T^{*}\right) \leq \bar{R}$. Now, we can extend and repeat this argument for a time step $\Delta t:=\frac{1}{2} \min \left(1, \bar{T}_{b}\right)$, where $\bar{T}_{b}=\frac{\bar{R}^{2-b}}{C_{b}(b-2)}$ if $b>2$ or $\bar{T}_{b}=+\infty$ if $b \leq 2$, obtaining a solution up to time $T^{*}+\Delta t$ such that $r_{2}(t) \leq \bar{R}$ for all $t \in\left[0, T^{*}+\Delta t\right]$. Since $\Delta t$ is independent of the initial data and $\varepsilon>0$, then we can extend the solution for all times.

Finally, the a priori estimates on the support of $\hat{\mu}$ show that the support of the solution remains compact for all times.

6.2. Proof of the Theorem 9. It is first convenient to rewrite 56 as:

$$
\psi_{a}(s)=\frac{\sigma_{N-1}}{\sigma_{N}} \int_{0}^{\pi} \frac{(1-s \cos \theta)(\sin \theta)^{N-2}}{\left(1+s^{2}-2 s \cos \theta\right)^{\frac{2-a}{2}}} d \theta .
$$

We recall that $\omega(r, \eta)$ is the velocity at $r$ generated by $\partial B(0, \eta)$. So a $\delta_{R}$ with $R>0$ is a steady state if and only if $\omega(R, R)=0$, i.e.

$$
R=R_{a b}=\left(\frac{\psi_{b}(1)}{\psi_{a}(1)}\right)^{\frac{1}{a-b}}
$$

where we have used (55).

Proof of the Theorem 9. The point (ii) is a direct consequence of Lemma 3, Let us prove (ii). From Lemma 3, see also [30] it is clear that $\omega \in C^{1}\left(\mathbb{R}_{+}^{2}\right)$ and we have

$$
\frac{\partial \omega}{\partial r}\left(R_{a b}, R_{a b}\right)=R_{a b}^{b-2}\left[(b-1) \psi_{b}(1)-\psi_{b}^{\prime}(1)\right]-R_{a b}^{a-2}\left[(a-1) \psi_{a}(1)-\psi_{a}^{\prime}(1)\right]
$$

After some algebra, one easily get from (61) and $(62)$ that $\frac{\partial \omega}{\partial r}\left(R_{a b}, R_{a b}\right)>0$ is equivalent to

$$
a-\frac{\psi_{a}^{\prime}(1)}{\psi_{a}(1)}<b-\frac{\psi_{b}^{\prime}(1)}{\psi_{b}(1)} \text {. }
$$


Both $\psi_{a}(1)$ and $\psi_{a}^{\prime}(1)$ can be expressed in terms of the Beta function. Recall that one of the expressions of the Beta function is:

$$
\beta(x, y)=2 \int_{0}^{\pi / 2}(\cos \theta)^{2 x-1}(\sin \theta)^{2 y-1} d \theta .
$$

We first compute $\psi_{a}(1)$. Using 60):

$$
\begin{aligned}
\frac{\sigma_{N}}{\sigma_{N-1}} \psi_{a}(1) & =\int_{0}^{\pi} \frac{(1-\cos \theta)}{A(1, \theta)^{2-a}}(\sin \theta)^{N-2} d \theta=2^{\frac{a-2}{2}} \int_{0}^{\pi}(1-\cos \theta)^{a / 2}(\sin \theta)^{N-2} d \theta \\
& =2^{\frac{a-2}{2}} \int_{0}^{\pi}\left(2 \sin ^{2} \frac{\theta}{2}\right)^{a / 2}\left(2 \cos \frac{\theta}{2} \sin \frac{\theta}{2}\right)^{N-2} d \theta \\
& =2^{a+N-3} \int_{0}^{\pi}\left(\sin \frac{\theta}{2}\right)^{a+N-2}\left(\cos \frac{\theta}{2}\right)^{N-2} d \theta \\
& =2^{a+N-3} \beta\left(\frac{a+N-1}{2}, \frac{N-1}{2}\right)
\end{aligned}
$$

where we have used the fact that $A(1, \theta)=\sqrt{2(1-\cos \theta})$ and the identities $1-\cos \theta=2 \sin ^{2} \frac{\theta}{2}$ and $\sin \theta=2 \cos \frac{\theta}{2} \sin \frac{\theta}{2}$. Similarly we compute

$$
\begin{aligned}
\frac{\sigma_{N}}{\sigma_{N-1}} \frac{N-1}{(a-2)(a+N-2)} \psi_{a}^{\prime}(1) & =\int_{0}^{\pi} \frac{(\sin \theta)^{N}}{A(1, \theta)^{4-a}} d \theta=\int_{0}^{\pi} \frac{(\sin \theta)^{N}}{(2(1-\cos \theta))^{\frac{4-a}{2}}} d \theta \\
& =\int_{0}^{\pi} \frac{\left(2 \cos \frac{\theta}{2} \sin \frac{\theta}{2}\right)^{N}}{\left(2\left(2 \sin ^{2} \frac{\theta}{2}\right)\right)^{\frac{4-a}{2}}} d \theta \\
& =2^{N+a-4} \int_{0}^{\pi}\left(\cos \frac{\theta}{2}\right)^{N}\left(\sin \frac{\theta}{2}\right)^{N+a-4} d \theta \\
& =2^{N+a-4} \beta\left(\frac{a+N-3}{2}, \frac{N+1}{2}\right)
\end{aligned}
$$

Note that since $a+N-3>0$ the Beta function is well defined. If we compute the quotient we obtain:

$$
\frac{\psi_{a}^{\prime}(1)}{\psi_{a}(1)}=\frac{1}{2} \frac{(a-2)(a+N-2)}{N-1} \frac{\beta\left(\frac{a+N-3}{2}, \frac{N+1}{2}\right)}{\beta\left(\frac{a+N-1}{2}, \frac{N-1}{2}\right)} .
$$

At this point, we remind that $\beta(z, t)=\frac{\Gamma(z) \Gamma(t)}{\Gamma(z+t)}$. With this expression, the quotient can be simplified as

$$
\frac{\psi_{a}^{\prime}(1)}{\psi_{a}(1)}=\frac{1}{2} \frac{(a-2)(a+N-2)}{N-1} \frac{\Gamma\left(\frac{N+1}{2}\right) \Gamma\left(\frac{a+N-3}{2}\right)}{\Gamma\left(\frac{a+N-1}{2}\right) \Gamma\left(\frac{N-1}{2}\right)},
$$

and if we use that $\Gamma(z+1)=z \Gamma(z)$ then, the gamma quotient can be reduced to $(N-1) /(a+N-3)$ and then we obtain

$$
\frac{\psi_{a}^{\prime}(1)}{\psi_{a}(1)}=\frac{1}{2} \frac{(a-2)(a+N-2)}{a+N-3} .
$$

Plugging the above expression into $\sqrt{63}$ ) and doing some algebra we deduce

$$
(a+N-3) b^{2}+\left(N^{2}-7 N+10-a^{2}\right) b-\left(N^{2}-7 N+10\right) a-(N-3) a^{2}>0 .
$$


The roots of the quadratic form are

$$
b=a \quad \text { and } \quad b=\frac{3 a-N a-10+7 N-N^{2}}{a+N-3}
$$

which gives (ii). To prove (iii) one just need to replace the $<\operatorname{sign}$ by a $>$ in $(63)$ to get the first condition. For the second condition of stability, we can easily compute $\frac{\partial \omega}{\partial \eta}$ from (55) and (62) to get

$$
\left(\frac{\partial \omega}{\partial r}+\frac{\partial \omega}{\partial \eta}\right)\left(R_{a b}, R_{a b}\right)=R_{a b}^{b-2}(b-1) \psi_{b}(1)-R_{a b}^{a-2}(a-1) \psi_{a}(1) .
$$

Now, using the definition of $R_{a b}$ in (61), we finally obtain

$$
\left(\frac{\partial \omega}{\partial r}+\frac{\partial \omega}{\partial \eta}\right)\left(R_{a b}, R_{a b}\right)=b-a<0 .
$$

The stated instability and stability are direct applications of Theorem 3 and Theorem 6 respectively.

Remark 12. We want to point out that the general theory developed in the previous sections is still working in dimension $N=1$ for even solutions which correspond to the radially symmetric solutions in higher dimensions. In the case $N=1$, and for even solutions, the function $\psi_{a}$, corresponding to $W(x)=\frac{|x|^{a}}{a}$ reads

$$
\psi_{a}(s)=\frac{1}{2}\left[(1-s)|1-s|^{a-1}+(1+s)|1+s|^{a-1}\right] .
$$

One can easily check that the properties of the function $\psi_{a}$ and $\omega$ for $W(x)=\frac{|x|^{a}}{a}-\frac{|x|^{b}}{b}$ in $N=1$ are the same as in Lemma 7 and in Theorem 9. The radius is $R_{a b}=\frac{1}{2}$ whatever the powers are, see (61). Theorem 9 applies: if $b \in(1,2)$ then we are in the instability case (i) and if $b \in[2, a)$ we are in the stability case. The curve which separates the instability and stability regions in Figure 1 degenerates and becomes the line $b=2$.

Moreover, in [33, 32 the authors proved the existence of weak solutions and convergence, up to extractions of subsequences, of $\rho(\cdot, t)$ for potentials like $W(x)=\frac{|x|^{2}}{2}-\frac{|x|^{b}}{b}, b \in(0,1]$. They also showed numerical simulations supporting the conjecture that the stationary state $\frac{1}{2}\left(\delta_{x=-1 / 2}+\delta_{x=1 / 2}\right)$ is unstable. Note that our Theorem 9, only applies in $N=1$ for $1<$ $b<a$. Since global existence was proven in [33, 32, then our instability result also applies in those cases. Summarizing, we can include $a=2, b \in(0,1]$ in the instability regions using Theorem 9 .

\section{Numerical RESUlts}

In this section, we illustrate the previous results and get some further conjectures for the instability cases. Our numerical code is based on the inverse distribution function in radial coordinates. As it was reminded in (31), the equation for the inverse distribution function reads

$$
\frac{\partial \varphi}{\partial t}(t, \xi)=\int_{0}^{1} \omega(\varphi(t, \xi), \varphi(t, \tilde{\xi})) d \tilde{\xi}
$$

A solution of (5) converges to a Dirac mass if and only if its pseudo inverse distribution becomes flat. 
Numerical codes based on (64) are then more stable when dealing with mass concentration. We will then use a backward Euler scheme in time coupled to a composite Simpson rule to approximate the integral term, and solve the resulting nonlinear system by the NewtonRaphson algorithm. Let us remark that the convergence of the semi-discrete backward Euler scheme is equivalent to the convergence of the JKO variational scheme for (1) (see [13, 22]). The convergence of the semi-discrete backward Euler scheme is therefore known under suitable conditions on the interaction potential, see [18] for details. All simulations are done for $N=2$.

Test case, total concentration at the origin: $W(x)=\frac{|x|^{2}}{2}$. In this case, (64) reduces to $\frac{\partial \varphi}{\partial t}(t, \xi)+\varphi(t, \xi)=0$. To test our scheme, we use this attractive potential for which the solution converges exponentially fast to a total concentration at zero, that is to $\bar{\varphi} \equiv 0$. See Figure 2,

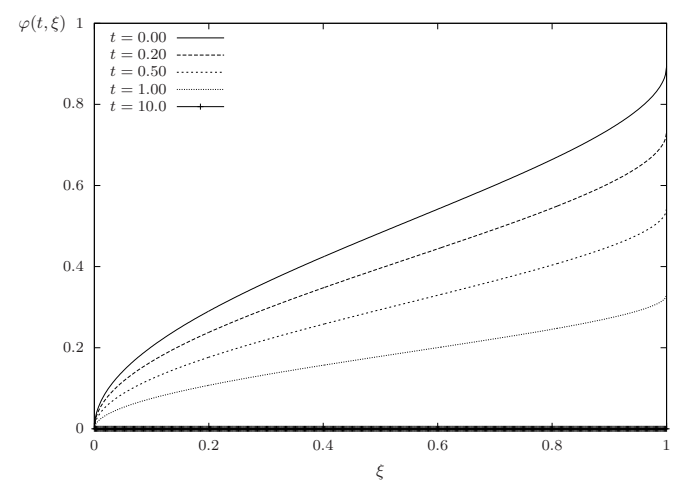

Figure 2. Evolution of $\xi \mapsto \varphi(t, \xi)$ for $W(x)=\frac{|x|^{2}}{2}$ towards total concentration at 0 .

Stability Case for the Spherical Shell: $W(x)=\frac{|x|^{4}}{4}-\frac{|x|^{2}}{2}$. In this case, we have an repulsive-attractive power law potential with powers in the stability region of Figure 1. We thus expect that the mass will concentrate towards a spherical shell, thanks to the results of Theorem 9. The radius of the spherical shell can be computed using (61):

$$
R_{a b}=\left(\frac{\psi_{2}(1)}{\psi_{4}(1)}\right)^{\frac{1}{2}}=\frac{\sqrt{3}}{3} .
$$

For $b \geq 2$ and both $a$ and $b$ integers, one can compute explicitly the expression for the velocity field $\omega(r, \eta)$, which is a polynomial function, in our case $\omega(r, \eta)=-r^{3}-2 r \eta^{2}+r$. The evolution of $\varphi$ is shown in Figure 3 . In Figure 3 we also plot the velocity field $r \mapsto \omega\left(r, R_{a b}\right)$. Notice that $r \mapsto \omega\left(r, R_{a b}\right)$ satisfies the conditions of Theorems 5 and $6 \quad \omega\left(R_{a b}, R_{a b}\right)=0$, $\partial_{1} \omega\left(R_{a b}, R_{a b}\right)<0, \operatorname{sign}\left(\omega\left(r, R_{a b}\right)\right)=\operatorname{sign}\left(R_{a b}-r\right), \partial_{1} \omega\left(0, R_{a b}\right)>0$.

Instability Case for the Spherical Shell: $W(x)=\frac{|x|^{2}}{2}-|x|$. In this case, the powers are in the instability region of Figure 1, below the curve $b=\frac{a}{a-1}$. Then, due to the results in Theorem 9, a spherical shell is unstable. One can notice on Figure 4 that the function $r \mapsto \omega\left(r, R_{a b}\right)$ associated to the potential $W(x)=\frac{|x|^{2}}{2}-|x|$ satisfies $\partial_{1} \omega\left(R_{a b}, R_{a b}\right)>0$, so that the instability condition of Theorem 3 is indeed satisfied. 

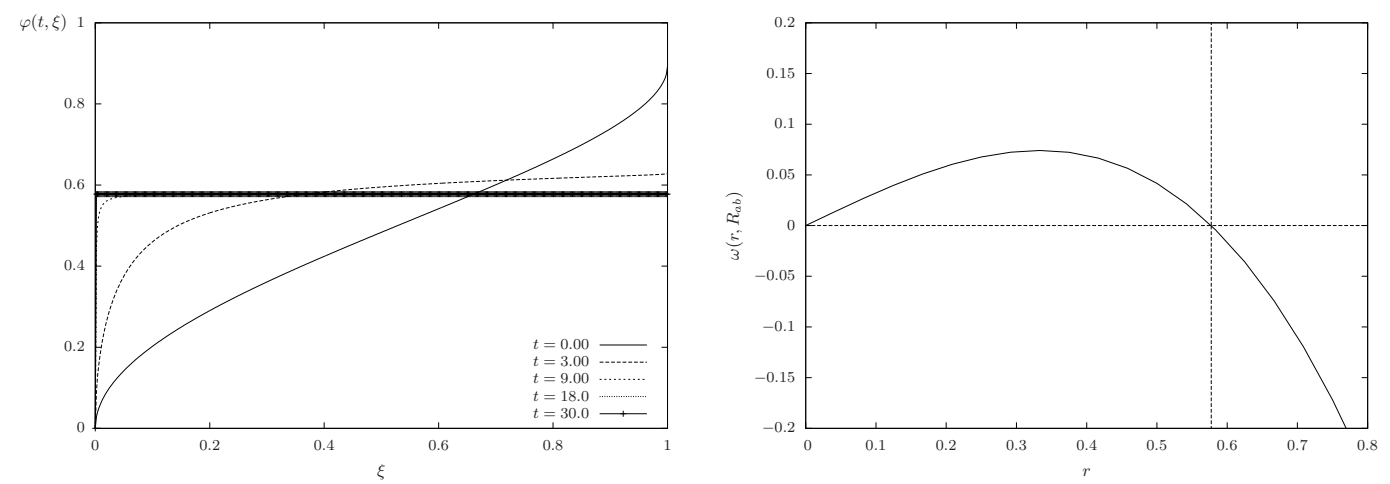

Figure 3. Case $W(x)=\frac{|x|^{4}}{4}-\frac{|x|^{2}}{2}$. Left: Evolution of $\xi \mapsto \varphi(t, \xi)$ towards the uniform distribution on the sphere of radius $R_{a b}=\frac{\sqrt{3}}{3}$. Right: Velocity field $r \mapsto \omega\left(r, \frac{\sqrt{3}}{3}\right)$ with the vertical line pointing out $R_{a b}$.
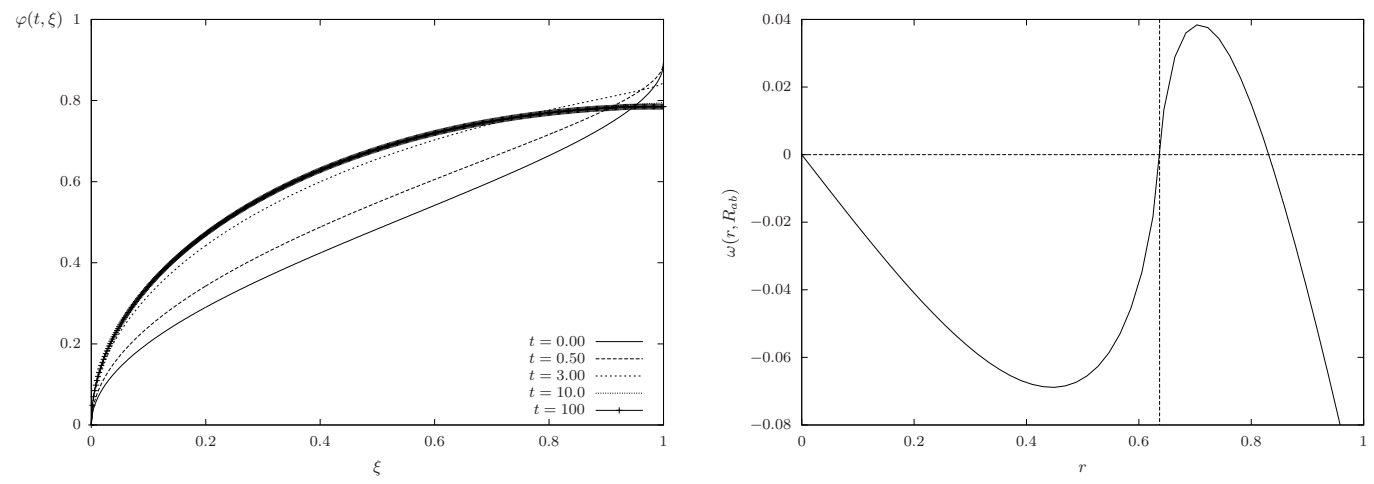

Figure 4. Case $W(x)=\frac{|x|^{2}}{2}-|x|$. Left: Evolution of $\xi \mapsto \varphi(t, \xi)$ towards a stationary profile, possibly an integrable function. Right: Velocity field $r \mapsto \omega\left(r, R_{a b}\right)$ with the vertical line pointing out $R_{a b} \sim 0.6366$.

Figure 4 shows that the solution seems to converge to some stationary state which does not have any singular part, i.e., possibly an integrable function. Numerically, this behavior appears for any powers $a, b$ in the instability region of Figure 1. We conjecture that in this region there exists integrable radial stationary states which are locally stable under radial perturbations. This has already been proved in the particular case of $b=2-N$ and $a \geq 2$ in 34]. Some numerical simulations using particle systems done in 39] however suggest that these stationary states might be unstable for non radial perturbations.

Energy dissipation. We remind that the energy functional is given by

$$
E[\rho](t)=\iint_{\mathbb{R}^{N} \times \mathbb{R}^{N}} W(x-y) \rho(t, x) \rho(t, y) d y d x .
$$


Using the polar change of coordinates $x=r \sigma$ and $y=s \tilde{\sigma}$ and using the radial symmetry of $\rho(t, \cdot)$, this energy writes:

$$
E[\hat{\rho}](t)=\frac{1}{2 \sigma_{N}} \iint_{\mathbb{R}_{+}^{2}} \int_{\partial B(0,1)} W\left(r \sigma-s e_{1}\right) \hat{\rho}(t, r) \hat{\rho}(t, s) d \sigma d s d r .
$$

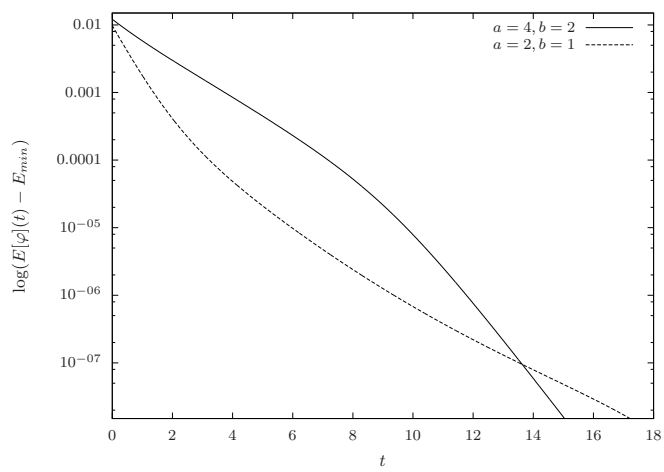

FiguRE 5. Energy decay in logarithmic scale for the regular repulsiveattractive potential, case $a=4$ and $b=2$ (solid line) and for the singular repulsive-attractive potential, case $a=2$ and $b=1$ (dashed line). Note that $E_{\text {min }}$ is the numerical limit of the energy as $t \rightarrow \infty$.

A formal calculation implies that the derivative w.r.t. time of the energy is negative and given by

$$
\frac{d}{d t} E[\hat{\rho}](t)=-\int_{\mathbb{R}^{+}} \hat{\rho}(t, r) \hat{v}(t, r)^{2} d r,
$$

the energy should then decrease in time. Using radially symmetric coordinates, the energy functional for the inverse distribution function is given by

$$
E[\varphi](t)=\frac{1}{2 \sigma_{N}} \int_{0}^{1} \int_{0}^{1} \int_{\partial B(0,1)} W\left(\varphi(t, \xi) \sigma-\varphi(t, \tilde{\xi}) e_{1}\right) d \sigma d \tilde{\xi} d \xi .
$$

We have computed the energy using the formula (65) to check numerically, in each case, that the energy decreases. In Figure 5 we observe the exponential decay of the energy for the two numerical examples presented above for repulsive-attractive potentials.

\section{Appendix}

Let us start by some differential geometry facts. For the sake of clarity, we first define the type of hypersurfaces we will work with.

Definition 5. $\mathcal{M} \subset \mathbb{R}^{N}$ is a $C^{2}$ hypersurface (manifold of dimension $N-1$ ) if for any $\bar{x} \in \mathcal{M}$ there exists a $C^{2}$ chart $(U, \varphi)$, i.e., a pair of an open connected set and a $C^{2}$ diffeomorphism $\varphi: U \longrightarrow \mathbb{R}^{N}$, with $\bar{x} \in U \subset \mathbb{R}^{N}$ such that $\varphi(\bar{x})=0$ and $y \in \mathcal{M} \cap U$ if and only if $\varphi(y) \in\{0\} \times \mathbb{R}^{N-1}$.

We will need some technical result from differential geometry in order to deal with the regularity of the function $\omega$ in (2) and its generalizations to any compact hypersurface. Note 
first that if $\mathcal{M} \subset \mathbb{R}^{N}$ is a hyperplane then $M \cap \partial B(x, r)$ is a $N-2$ dimensional sphere of radius $\left(r^{2}-\operatorname{dist}(x, \mathcal{M})^{2}\right)_{+}^{1 / 2}$ and therefore its surface area is

$$
|\mathcal{M} \cap \partial B(x, r)|_{\mathcal{H}^{N-2}}=\sigma_{N-1}\left(r^{2}-\operatorname{dist}(x, \mathcal{M})^{2}\right)_{+}^{\frac{N-2}{2}}
$$

where $\mathcal{H}^{d}$ is the d-dimensional Hausdorff measure, and we remind that $\sigma_{N-1}$ is the surface area of the unit sphere in $\mathbb{R}^{N-1}$.

The following result is a classical consequence of uniform graphs lemmas in differential geometry. They state that a compact regular hypersurface can be covered by graphs with bounds on their derivatives depending only on the uniform bound of the second fundamental form. We refer to [50, Lemma 4.1.1]. This allows to show that the volume elements locally converge to those of a hyperplane in a uniform manner.

Lemma 8. Let $\mathcal{M} \subset \mathbb{R}^{N}$ be a $C^{2}$ compact hypersurface of dimension $N-1$ immersed in $\mathbb{R}^{N}$. Then there exist small enough $r_{0}>0$ and constants $C, \tilde{C}>0$ depending on the global bound of the second fundamental form of $\mathcal{M}$ such that for all $0<r \leq r_{0}$, and all $x \in \mathbb{R}^{N}$ with $\operatorname{dist}(x, \mathcal{M})<r_{0}$

$$
\tilde{C}\left(r^{2}-\operatorname{dist}(x, \mathcal{M})^{2}\right)_{+}^{\frac{N-2}{2}} \leq|\mathcal{M} \cap \partial B(x, r)|_{\mathcal{H}^{N-2}} \leq C\left(r^{2}-\operatorname{dist}(x, \mathcal{M})^{2}\right)_{+}^{\frac{N-2}{2}} .
$$

Remark 13. Let us note that the previous Lemma is trivial in the case of $\mathcal{M}=\partial B(0, \eta)$ for any $\eta>0$ since the intersection of two $(N-1)$-dimensional spheres of different radius is always a $(N-2)$-dimensional sphere lying on a hyperplane. In fact, we can easily compute that if two spheres $\partial B(0, \eta)$ and $\partial B(x, r)$ intersect, that is ||$x|-\eta| \leq r$, then

$$
|\partial B(0, \eta) \cap \partial B(x, r)|_{\mathcal{H}^{N-2}}=\sigma_{N-1} r_{1}^{N-2}
$$

where $r_{1}=r_{1}(\eta, r, \operatorname{dist}(x, \partial B(0, \eta)))$ is the radius of the intersection, which is computable:

$$
r_{1}=\eta \sqrt{1-\left(\frac{|x|^{2}+\eta^{2}-r^{2}}{2|x| \eta}\right)^{2}} \sim \sqrt{\frac{\eta}{|x|}} \sqrt{r^{2}-\operatorname{dist}(x, \partial B(0, \eta))^{2}},
$$

as $r-\operatorname{dist}(x, \partial B(0, \eta)) \rightarrow 0$. The constants $r_{0}, C$, and $\tilde{C}$ of Lemma 13 can then be taken uniform for variations of the radius in bounded intervals, i.e., for $0<\eta_{1}<\eta<\eta_{2}$.

We now can deal with the continuity of the velocity fields generated by probability densities concentrated on manifolds. Recall that $\mathbb{R}_{+}=(0,+\infty)$.

Lemma 9. Let $\mathcal{M} \subset \mathbb{R}^{N}$ be a compact $C^{2}$ hypersurface, $\mu$ a probability distribution such that $\bar{\mu}=\phi \delta_{\mathcal{M}}$, where $\phi \in L^{\infty}(\mathcal{M})$, and $g \in C\left(\mathbb{R}^{N} /\{0\}\right)$ a radially symmetric function which is locally integrable on hypersurfaces. Then, the function

$$
v(x)=\int_{\mathbb{R}^{N}} g(x-y) d \bar{\mu}(y)
$$

is continuous in $x \in \mathbb{R}^{N}$. Moreover, the same results hold while replacing $g(x)$ by a nonradially symmetric function $G \in C\left(\mathbb{R}^{N} /\{0\}\right)$ such that $|G(x)| \leq|g(x)|$, where $g$ satisfies the properties above.

Proof. It is straightforward to check that $v(x)$ is continuous for all $\bar{x} \notin \mathcal{M}$. Let $\bar{x} \in \mathcal{M}$ and let $r_{0}$ be given by Lemma 8 . For $0<\varepsilon<r_{0}$, let $\chi_{\varepsilon} \in C^{\infty}\left(\mathbb{R}_{+}\right)$be a cut-off function, such 
that $\chi_{\varepsilon}=1$ on $[0, \varepsilon / 2]$, and $\chi_{\varepsilon}=0$ on $[\varepsilon, \infty)$. The function $v$ can then be written as

$$
\begin{aligned}
v(x) & =\int_{\mathcal{M}} g(x-y) \chi_{\varepsilon}(x-y) d \bar{\mu}(y)+\int_{\mathcal{M}} g(x-y)\left[1-\chi_{\varepsilon}(x-y)\right] d \bar{\mu}(y) \\
& :=v_{1}^{\varepsilon}(x)+v_{2}^{\varepsilon}(x) .
\end{aligned}
$$

It is clear that $v_{2}^{\varepsilon}$ is continuous on $x \in \mathbb{R}^{N}$, since $g$ is continuous away from the origin and $\operatorname{supp}(\bar{\mu})=\mathcal{M}$ is compact. Moreover, given the set $U=\left\{x \in \mathbb{R}^{N}: \operatorname{dist}(x, \mathcal{M})<r_{0}\right\}$, we can estimate for all $x \in U$

$$
\begin{aligned}
\left|v_{1}^{\varepsilon}(x)\right| & \leq \int_{\mathbb{R}^{N}}|g(x-y)| \chi_{\varepsilon}(x-y) d \bar{\mu}(y) \leq \int_{B(x, \varepsilon)}|g(x-y)| d \bar{\mu}(y) \\
& \leq\|\phi\|_{L^{\infty}(\mathcal{M})} \int_{0}^{\varepsilon}|\hat{g}(r)||\{y \in \mathcal{M} ;|y-x|=r\}|_{\mathcal{H}^{N-2}} d r \leq C\|\phi\|_{L^{\infty}(\mathcal{M})} \int_{0}^{\varepsilon}|\hat{g}(r)| r^{N-2} d r
\end{aligned}
$$

where (66) is used. Moreover, by construction $v_{1}^{\varepsilon}(x)=0$ for all $x \notin U$ for $\varepsilon<r_{0}$. Therefore, due to the integrability over hypersurfaces of $g$, then

$$
\lim _{\varepsilon \rightarrow 0}\left\|v_{1}^{\varepsilon}\right\|_{L^{\infty}\left(\mathbb{R}^{N}\right)}=0 .
$$

This is enough to show the continuity of $v$ on $\mathcal{M}$ : for any $\delta>0$, there exists $\varepsilon>0$ such that $\left\|v_{1}^{\varepsilon}\right\|_{L^{\infty}\left(\mathbb{R}^{N}\right)} \leq \frac{\delta}{2}$. Since $v_{2}^{\varepsilon}$ is continuous, there exists $\kappa>0$ such that $\left|v_{2}^{\varepsilon}(x)-v_{2}^{\varepsilon}(\bar{x})\right| \leq \frac{\delta}{2}$ if $|x-\bar{x}| \leq \kappa$. Then, $|v(x)-v(\bar{x})| \leq \delta$ if $|x-\bar{x}| \leq \kappa$. The last part of the proof is an adaptation of the previous arguments since the integral inside the norm is less or equal than $v_{1}^{2 \epsilon}$.

Now, we want to obtain the continuity with respect to the hypersurface for the velocity fields associated to measures concentrated on them. We restrict to the case of spheres since we only need this particular case. The proof uses the transport distance $d_{\infty}$. We remind the reader that it is introduced in Section 3.

Lemma 10. Let $\mathcal{M}_{\eta}:=\partial B(0, \eta)$ and $\bar{\mu}_{\eta}=\phi_{\eta} \delta_{\mathcal{M}_{\eta}}$ be probability measures such that $\phi_{\eta} \in$ $L^{\infty}\left(\mathcal{M}_{\eta}\right)$ with $0<\eta$. Let $g \in C^{1}\left(\mathbb{R}^{N} \backslash\{0\}\right)$ be a radially symmetric function which is locally integrable on hypersurfaces. If the functions $\phi_{\eta}$ are uniformly bounded in $\eta$ and $d_{\infty}\left(\bar{\mu}_{\eta}, \bar{\mu}_{\tilde{\eta}}\right) \rightarrow$ 0 as $\eta-\tilde{\eta} \rightarrow 0$, then

$$
v(x, \eta)=\int_{\partial B(0, \eta)} g(x-y) d \bar{\mu}_{\eta}(y)
$$

is continuous in $\mathbb{R}^{N} \times \mathbb{R}_{+}$. Moreover, the same result holds while replacing $g(x)$ by a nonradially symmetric function $G \in C^{1}\left(\mathbb{R}^{N} \backslash\{0\}\right)$ such that $|G(x)| \leq|g(x)|$ with the properties above.

Proof. Lemma 9 implies directly the continuity with respect to $x$ for all fixed $\eta$. Using the Remark 13 and the proof of Lemma 9, it can be easily checked that this continuity in $x$ is uniform in $\eta$. Indeed $\left|v_{1}^{\epsilon}\right|$ can be made small uniformly in $\eta$ and, due to the estimate $\left|\nabla v_{2}^{\epsilon}(x)\right| \leq \sup _{\partial B(x, \eta)}\left|\nabla\left[g\left(1-\chi_{\epsilon}\right)\right]\right|, v_{2}^{\epsilon}$ is continuous uniformly in $\eta$. Therefore, we only need to show the continuity in $\eta$ of $v$ for a fixed $x \in \mathbb{R}^{N}$.

As in the proof of Lemma 9 , let $r_{0}$ be as obtained in Remark 13 uniform in $0<\eta_{1}<\eta<\eta_{2}$. We choose again $0<\varepsilon<r_{0}$ and $\chi_{\epsilon} \in C^{\infty}\left(\mathbb{R}_{+}\right)$a cut-off function, such that $\chi_{\varepsilon}=1$ on $[0, \varepsilon / 2]$, and $\chi_{\varepsilon}=0$ on $[\varepsilon, \infty)$. We can write $v(x, \eta)=v_{1}^{\varepsilon}(x, \eta)+v_{2}^{\varepsilon}(x, \eta)$ analogously to (67). As in Lemma 9 using the properties of $g$ and the uniformity in Remark (13), we can easily show that

$$
\lim _{\varepsilon \rightarrow 0}\left\|v_{1}^{\varepsilon}(\cdot, \eta)\right\|_{L^{\infty}\left(\mathbb{R}^{N}\right)}=0
$$


uniformly in $0<\eta_{1}<\eta<\eta_{2}$. Therefore, for any $\delta>0$, there exists $r_{0}>\varepsilon>0$ such that $\left\|v_{1}^{\varepsilon}(\cdot, \eta)\right\|_{L^{\infty}\left(\mathbb{R}^{N}\right)} \leq \frac{\delta}{4}$ uniformly in $0<\eta_{1}<\eta<\eta_{2}$. Now, we estimate

$$
\begin{aligned}
|v(x, \eta)-v(x, \tilde{\eta})| \leq & \left\|v_{1}^{\varepsilon}(\cdot, \eta)\right\|_{L^{\infty}\left(\mathbb{R}^{N}\right)}+\left\|v_{1}^{\varepsilon}(\cdot, \tilde{\eta})\right\|_{L^{\infty}\left(\mathbb{R}^{N}\right)} \\
& +\left|\int_{\mathbb{R}^{N}} g(x-y)\left[1-\chi^{\varepsilon}(|x-y|)\right] d\left(\bar{\mu}_{\eta}-\bar{\mu}_{\tilde{\eta}}\right)(y)\right| \\
\leq & \frac{\delta}{2}+\left\|\nabla\left[g\left(1-\chi^{\varepsilon}\right)\right]\right\|_{L^{\infty}(\Delta)} d_{\infty}\left(\bar{\mu}_{\eta}, \bar{\mu}_{\tilde{\eta}}\right),
\end{aligned}
$$

where $\Delta$ is the convex hull of the set $\{x\}-\left(\operatorname{supp} \bar{\mu}_{\eta}\right) \cup\left(\operatorname{supp} \bar{\mu}_{\tilde{\eta}}\right)$. Notice that the set $\Delta$ is uniformly bounded in $\eta$ and $\tilde{\eta}$.

This estimate shows the continuity in $\eta$ since $d_{\infty}\left(\bar{\mu}_{\eta}, \bar{\mu}_{\tilde{\eta}}\right) \rightarrow 0$ as $\eta \rightarrow \tilde{\eta}$, and thus, the last term is bounded by $\delta / 2$ provided that $\eta$ is close enough to $\tilde{\eta}$. Again, the final part of this Lemma is a small variation of the previous arguments.

Finally, we complete the results by showing that if the function is not locally integrable on hypersurfaces then the velocity field is not bounded.

Lemma 11. Let $\mathcal{M}_{\eta}:=\partial B(0, \eta)$ and $\bar{\mu}_{\eta}=\phi_{\eta} \delta_{\mathcal{M}_{\eta}}$ be probability measures such that $\phi_{\eta}(x) \geq$ $\phi_{0}>0$ for all $\eta_{1} \leq \eta \leq \eta_{2}$. Let $g \in C\left(\mathbb{R}^{N} \backslash\{0\}\right)$ be a nonnegative radially symmetric function which is not locally integrable on hypersurfaces. Then For all $M>0$ there exists $\delta>0$ such that

$$
\operatorname{dist}\left(x, \mathcal{M}_{\eta}\right)<\delta \Longrightarrow \int_{\mathbb{R}^{N}} g(x-y) d \bar{\mu}_{\eta}(y) \geq M \quad \text { for all } x \in \mathbb{R}^{N} \text { and for all } \eta_{1} \leq \eta \leq \eta_{2} \text {. }
$$

Proof. Using Lemma 8 and Remark 13 , for $x \in \mathbb{R}^{N}$ with $\operatorname{dist}\left(x, \mathcal{M}_{\eta}\right)<r_{0}$, we get

$$
\begin{aligned}
\int_{\mathbb{R}^{N}} g(x-y) d \bar{\mu}_{\eta}(y) & \geq \int_{|x-y|<r_{0}} g(x-y) d \bar{\mu}_{\eta}(y) \geq \phi_{0} \int_{0}^{r_{0}} \hat{g}(r)\left|\left\{y \in \mathcal{M}_{\eta} ;|y-x|=r\right\}\right|_{\mathcal{H}^{N-2}} d r \\
& \geq \phi_{0} \tilde{C} \int_{0}^{r_{0}} \hat{g}(r)\left(r^{2}-\operatorname{dist}\left(x, \mathcal{M}_{\eta}\right)^{2}\right)_{+}^{\frac{N-2}{2}} d r .
\end{aligned}
$$

Since $\int_{0}^{1} \hat{g}(r) r^{N-2} d r=+\infty$ and $g$ is continuous and nonnegative on $(0,1]$, we deduce that

$$
\lim _{\operatorname{dist}\left(x, \mathcal{M}_{\eta}\right) \rightarrow 0} \int_{0}^{r_{0}} \hat{g}(r)\left(r^{2}-\operatorname{dist}\left(x, \mathcal{M}_{\eta}\right)^{2}\right)_{+}^{\frac{N-2}{2}} d r=+\infty,
$$

by the monotone convergence theorem, which conclude the proof.

Acknowledgments. DB and JAC were supported by the projects Ministerio de Ciencia e Innovación MTM2011-27739-C04-02 and 2009-SGR-345 from Agència de Gestió d'Ajuts Universitaris i de Recerca-Generalitat de Catalunya. GR was supported by Award No. KUK-I1007-43 of Peter A. Markowich, made by King Abdullah University of Science and Technology (KAUST). DB, JAC and GR acknowledge partial support from CBDif-Fr ANR-08-BLAN0333-01 project. TL acknowledges the support from NSF Grant DMS-1109805. The authors warmly thank Joaquín Pérez in helping them with the differential geometry question related to Lemma 8 . 
D. BALAGUÉ ${ }^{1}$, J. A. CARRILLO ${ }^{2}$, T. LAURENT ${ }^{3}$ AND G. RAOUL 4

\section{REFERENCES}

[1] L. Ambrosio, E. Mainini, and S. Serfaty. Gradient flow of the chapman-rubinstein-schatzman model for signed vortices. preprint, 2010.

[2] L. Ambrosio and S. Serfaty. A gradient flow approach to an evolution problem arising in superconductivity. Communications on Pure and Applied Mathematics, LXI:1495-1539, 2008.

[3] L. A. Ambrosio, N. Gigli, and G. Savarè. Gradient flows in metric spaces and in the space of probability measures. Lectures in Mathematics. Birkhäuser, 2005.

[4] D. Balagué and J. A. Carrillo. Aggregation equation with growing at infinity attractive-repulsive potentials. to appear in Proceedings of HYP2010.

[5] A. B. T. Barbaro, K. Taylor, P. F. Trethewey, L. Youseff, and B. Birnir. Discrete and continuous models of the dynamics of pelagic fish: application to the capelin. Math. Comput. Simulation, 79(12):3397-3414, 2009.

[6] A. Bernoff and T. Chad. A primer of swarm equilibria. preprint.

[7] A. Bertozzi and J. Brandman. Finite-time blow-up of $l^{\infty}$-weak solutions of an aggregation equation. Comm. Math. Sci., 8(1):45-65, 2010. Special issue in honor of Andrew Majda's 60th birthday.

[8] A. Bertozzi, J. A. Carrillo, and T. Laurent. Blowup in multidimensional aggregation equations with mildly singular interaction kernels. Nonlinearity, 22:683-710, 2009.

[9] A. Bertozzi, J. Garnett, and T. Laurent. Characterization of radially symmetric finite time blowup in multidimensional aggregation equations. preprint.

[10] A. Bertozzi and T. Laurent. Finite-time blow-up of solutions of an aggregation equation in $\mathbb{R}^{n}$. Comm. Math. Phys., 274:717-735, 2007.

[11] A. Bertozzi, T. Laurent, and J. Rosado. Lp theory for the multidimensional aggregation equation. Comm. Pure Appl. Math., 64(1):45-83, 2011.

[12] B. Birnir. An ODE model of the motion of pelagic fish. J. Stat. Phys., 128(1 - 2):535-568, 2007.

[13] A. Blanchet, V. Calvez, and J. A. Carrillo. Convergence of the mass-transport steepest descent scheme for the subcritical Patlak-Keller-Segel model. SIAM J. Numer. Anal., 46(2):691-721, 2008.

[14] A. Blanchet, J. A. Carrillo, and N. Masmoudi. Infinite time aggregation for the critical patlak-keller-segel model in $\mathbb{R}^{2}$. Comm. Pure Appl. Math., 61(10):1449-1481, 2008.

[15] A. Blanchet, J. Dolbeault, and B. Perthame. Two-dimensional Keller-Segel model: optimal critical mass and qualitative properties of the solutions. Electron. J. Differential Equations, 44, 2006. 32 pp. (electronic).

[16] J.A. Cañizo, J.A. Carrillo, and J. Rosado. A well-posedness theory in measures for some kinetic models of collective motion. Math. Mod. Meth. Appl. Sci., 21:515-539, 2011.

[17] J. A. Carrillo, M. Di Francesco, A. Figalli, T. Laurent, and D. Slepčev. Confinement in nonlocal interaction equations. to appear in Nonlinear Analysis TMA, 2011.

[18] J. A. Carrillo, M. Di Francesco, A. Figalli, T. Laurent, and D. Slepčev. Global-in-time weak measure solutions and finite-time aggregation for nonlocal interaction equations. Duke Math. J., 156:229-271, 2011.

[19] J. A. Carrillo, M. P. Gualdani, and G. Toscani. Finite speed of propagation in porous media by mass transportation methods. C. R. Math. Acad. Sci. Paris, 338(10):815-818, 2004.

[20] J. A. Carrillo, R. J. McCann, and C. Villani. Kinetic equilibration rates for granular media and related equations: entropy dissipation and mass transportation estimates. Rev. Mat. Iberoamericana, 19(3):9711018, 2003.

[21] J. A. Carrillo, R. J. McCann, and C. Villani. Contractions in the 2-Wasserstein length space and thermalization of granular media. Arch. Ration. Mech. Anal., 179(2):217-263, 2006.

[22] J. A. Carrillo and J. S. Moll. Numerical simulation of diffusive and aggregation phenomena in nonlinear continuity equations by evolving diffeomorphisms. SIAM J. Sci. Comput., 31(6):4305-4329, 2009/10.

[23] J. A. Carrillo and J. Rosado. Uniqueness of bounded solutions to aggregation equations by optimal transport methods. In European Congress of Mathematics, pages 3-16. Eur. Math. Soc., Zürich, 2010.

[24] J.A. Carrillo, M.R. D'Orsogna, and V. Panferov. Double milling in self-propelled swarms from kinetic theory. Kin. Rel. Mod., 2:363-378, 2009.

[25] J.A. Carrillo, M. Fornasier, J. Rosado, and G. Toscani. Asymptotic flocking dynamics for the kinetic cucker-smale model. SIAM J. Math. Anal., 42:218-236, 2010.

[26] F. Cucker and S. Smale. Emergent behavior in flocks. IEEE Trans. Automat. Control, 52(5):852-862, 2007. 
[27] F. Cucker and S. Smale. On the mathematics of emergence. Jpn. J. Math., 2(1):197-227, 2007.

[28] M. Pulvirenti D. Benedetto, E. Caglioti. A kinetic a kinetic equation for granular media. RAIRO Modél. Math. Anal. Numér., 31:615-641, 1997.

[29] J. Dolbeault and B. Perthame. Optimal critical mass in the two-dimensional Keller-Segel model in $\mathbb{R}^{2}$. $C$. R. Math. Acad. Sci. Paris, 339:611-616, 2004.

[30] H. Dong. The aggregation equation with power-law kernels: ill-posedness, mass concentration and similarity solutions. Communications in Mathematical Physics, 304:649-664, 2011.

[31] Q. Du and P. Zhang. Existence of weak solutions to some vortex density models. Siam J. Math. Anal., 34(6):1279-1299, 2003.

[32] K. Fellner and G. Raoul. Stability of stationary states of non-local equations with singular interaction potentials. to appear in Math. Comput. Modelling.

[33] K. Fellner and G. Raoul. Stable stationary states of non-local interaction equations. to appear in Math. Models Methods Appl. Sci., 2010.

[34] R. C. Fetecau, Y. Huang, and T. Kolokolnikov. Swarm dynamics and equilibria for a nonlocal aggregation model. to appear in Nonlinearity, 2011.

[35] E. Geigant, K. Ladizhansky, and A. Mogilner. An integrodifferential model for orientational distributions of f-actin in cells. SIAM Journal on Applied Mathematics, 59(3):787-809, 1998.

[36] L. Gosse and G. Toscani. Lagrangian numerical approximations to one-dimensional convolution-diffusion equations. SIAM J. Sci. Comput., 28(4):1203-1227 (electronic), 2006.

[37] S. Y. Ha and E. Tadmor. From particle to kinetic and hydrodynamic descriptions of flocking. Kinet. Relat. Models, 1(3):415-435, 2008.

[38] K. Kang, B. Perthame, A. Stevens, and J.J.L. Velazquez. An integro-differential equation model for alignment and orientational aggregation. J. Differential Equations, 264(4):1387-1421, 2009.

[39] T. Kolokonikov, H. Sun, D. Uminsky, and A. Bertozzi. Stability of ring patterns arising from 2d particle interactions. Physical Review E, 84(1):015203, 2011.

[40] T. Laurent. Local and global existence for an aggregation equation. Comm. Partial Differential Equations, 32(10-12):1941-1964, 2007.

[41] H. Li and G. Toscani. Long-time asymptotics of kinetic models of granular flows. Arch. Ration. Mech. Anal., 172:407-428, 2004.

[42] F. Lin and P. Zhang. On the hydrodynamic limit of ginzburg-landau vortices. Discrete Contin. Dynam. Systems, 6:121-142, 2000.

[43] E. Mainini. A global uniqueness result for an evolution problem arising in superconductivity. Bollettino dell unione matematica italiana, 2009.

[44] N. Masmoudi and P. Zhang. Global solutions to vortex density equations arising from sup-conductivity. Annales de l'Institut Henri Poincare, 22:441-458, 2005.

[45] R. J. McCann. Stable rotating binary stars and fluid in a tube. Houston J. Math., 32(2):603-631, 2006.

[46] A. Mogilner and L. Edelstein-Keshet. A non-local model for a swarm. J. Math. Biol., 38(6):534-570, 1999.

[47] A. Mogilner, L. Edelstein-Keshet, L. Bent, and A. Spiros. Mutual interactions, potentials, and individual distance in a social aggregation. J. Math. Biol., 47(4):353-389, 2003.

[48] J. Nieto, F. Poupaud, and J. Soler. High-field limit for the Vlasov-Poisson-Fokker-Planck system. Arch. Ration. Mech. Anal., 158(1):29-59, 2001.

[49] A. Okubo and Levin S.A. Diffusion and Ecological Problems, chapter 7, pages 197-237. Interdisciplinary Applied Mathematics: Mathematical Biology. Springer, New York, 2001, 2nd edition, 2001.

[50] Joaquín Pérez and Antonio Ros. Properly embedded minimal surfaces with finite total curvature. In The global theory of minimal surfaces in flat spaces (Martina Franca, 1999), volume 1775 of Lecture Notes in Math., pages 15-66. Springer, Berlin, 2002.

[51] I. Primi, A. Stevens, and J. J.L. Velazquez. Mass-selection in alignment models with non-deterministic effects. Comm. Partial Differential Equations, 34(5), 2009.

[52] G. Raoul. Non-local interaction equations: Stationary states and stability analysis. preprint, 2010.

[53] E. Sandier and S. Serfaty. A rigorous derivation of a free-boundary problem arising in superconductivity. Ann. Sci. École Norm. Sup., 33(4):561-592, 2000.

[54] E. Sandier and S. Serfaty. Vortices in the magnetic Ginzburg-Landau model. Progress in Nonlinear Differential Equations and their Applications, 70. Birkhäuser Boston Inc., 2007.

[55] C. Topaz, A. Bernoff, S. S. Logan, and W. Toolson. A model for rolling swarms of locusts. Eur. Phys. J. Special Topics, 157:93-109, 2008. 
[56] C. M. Topaz and A. L. Bertozzi. Swarming patterns in a two-dimensional kinematic model for biological groups. SIAM J. Appl. Math., 65:152-174, 2004.

[57] C. M. Topaz, A. L. Bertozzi, and M. A. Lewis. A nonlocal continuum model for biological aggregation. Bulletin of Mathematical Biology, 68(7):1601-1623, 2006.

[58] G. Toscani. One-dimensional kinetic models of granular flows. RAIRO Modél. Math. Anal. Numér., 34(6):1277-1291, 2000.

[59] C. Villani. Topics in optimal transportation, volume 58 of Graduate Studies in Mathematics. American Mathematical Society, Providence, RI, 2003.

[60] J. von Brecht, D. Uminsky, T. Kolokolnikov, and A. Bertozzi. Predicting pattern formation in particle interactions. in preparation.

[61] E. Weinan. Dynamics of vortex liquids in ginzburg-landau theories with applications to superconductivity. Physical Review B, 50(2):1126-1135, 1994.

1 Departament de Matemàtiques, Universitat Autònoma de Barcelona, E-08193 Bellaterra, SPAIN. E-MAIL: dbalague@mat.uab.cat.

2 ICREA and Departament de Matemàtiques, Universitat Autònoma de Barcelona, E-08193 Bellaterra, Spain. E-MAil: carrillo@mat.uab.es. On leave from: Department of Mathematics, Imperial College London, London SW7 2AZ, UK.

3 Department of Mathematics, University of California - Riverside, Riverside, CA 92521, USA. E-MAIL: laurent@math.ucr.edu.

${ }^{4}$ DAMTP, University of Cambridge, Wilberforce road, CB3 0WA, United Kingdom. E-mail: g.raoul@damtp.cam.ac.uk. 